\title{
Heterotic moduli stabilization with fractional Chern-Simons invariants
}

\author{
Sergei Gukov, ${ }^{1, *}$ Shamit Kachru, ${ }^{2,3, \dagger}$ Xiao Liu, ${ }^{2,3, *}$ and Liam McAllister ${ }^{2, \S}$ \\ ${ }^{1}$ Department of Physics, Harvard University, Cambridge, Massachusetts 02138, USA \\ ${ }^{2}$ Department of Physics, Stanford University, Stanford, California 94305, USA \\ ${ }^{3}$ Stanford Linear Accelerator Center, Stanford University, Menlo Park, California 94309, USA
}

(Received 11 November 2003; published 29 April 2004)

\begin{abstract}
We show that fractional flux from Wilson lines can stabilize the moduli of heterotic string compactifications on Calabi-Yau threefolds. We observe that the Wilson lines used in GUT symmetry breaking naturally induce a fractional flux. When combined with a hidden-sector gaugino condensate, this generates a potential for the complex structure moduli, Kähler moduli, and dilaton. This potential has a supersymmetric AdS minimum at moderately weak coupling and large volume. Notably, the necessary ingredients for this construction are often present in realistic models. We explore the type IIA dual phenomenon, which involves Wilson lines in D6branes wrapping a three-cycle in a Calabi-Yau threefold, and comment on the nature of the fractional instantons that change the Chern-Simons invariant.
\end{abstract}

DOI: $10.1103 /$ PhysRevD.69.086008

PACS number(s): 11.25.Mj

\section{INTRODUCTION}

When string theory is compactified on a Calabi-Yau manifold [1], the resulting low-energy field theory typically contains some number of massless scalar fields, or moduli. Gravitational experiments and the requirement of consistency with nucleosynthesis place rather strong constraints on the existence of such fields (see, e.g., Ref. [2]). If moduli were an essential feature of all string compactifications then model building would be very difficult. Fortunately, moduli are only endemic in the simplest, most symmetric constructions. General backgrounds involving fluxes, as well as nonperturbative effects, tend to create potentials for some or all moduli. Even so, although compactifications with reduced moduli spaces are easy to construct, it remains challenging to eliminate all of the moduli in a given model.

Two fields that have proven particularly difficult to stabilize are the Calabi-Yau volume and, in heterotic compactifications, the dilaton. The problem is especially acute in these cases because the dilaton and volume directly influence the gauge and gravitational couplings in our world, making rolling values unacceptable. Moreover, as these parameters govern the string and sigma-model perturbation expansions, a controllable compactification requires that the dilaton and volume be stabilized at weak coupling and large radius.

We will demonstrate that this can be achieved in a certain class of heterotic compactifications on Calabi-Yau spaces with a large fundamental group. The context for this proposal is the original work [3] of Dine, Rohm, Seiberg, and Witten, who observed that the combination of a gaugino condensate (in the hidden sector of the $E_{8} \times E_{8}$ heterotic string) and a background three-form flux generates a potential for the di-

\footnotetext{
*Electronic mail: gukov@ @omonaga.harvard.edu

†Electronic mail: skachru@stanford.edu

*Electronic mail: liuxiao@itp.stanford.edu

${ }^{\S}$ Electronic mail: 1pm@itp.stanford.edu
}

laton but leaves the cosmological constant zero at tree level. ${ }^{1}$ As was understood there and in more detail in subsequent work, because of the quantization condition for the threeform of the heterotic theory, the dilaton cannot be fixed at weak coupling. The essential difficulty is that the gaugino condensate term is nonperturbatively small when the coupling is weak, whereas quantization forces the flux term to be of order one. The resulting potential drives the dilaton to strong coupling.

It is important, however, that the Chern-Simons contribution to the heterotic three-form flux does not obey the same quantization condition as the contribution from the field strength of the antisymmetric tensor. In fact, as we will explain below, the Chern-Simons contribution of a flat gauge bundle can take fractional values of order $1 / N$, where $N$ is related to the order of the fundamental group. On Calabi-Yau manifolds with a sufficiently large fundamental group this provides a natural mechanism to stabilize the dilaton at weak coupling. The same effect stabilizes all Kähler moduli once the dependence of the gauge coupling on these moduli is correctly incorporated. For related earlier work see Refs. [5-11].

The requirements that the Calabi-Yau manifold should have non-trivial fundamental group and that the gauge bundle should have nonzero Wilson lines are actually well motivated by other model-building considerations. In fact, most models of particle physics based on Calabi-Yau compactifications of the heterotic string involve manifolds with nontrivial fundamental group and associated gauge bundles with Wilson lines.

A standard way to construct such manifolds is to quotient a simply-connected Calabi-Yau space by a freely acting discrete symmetry group $G$. The resulting string GUT model solves a number of important problems. For instance, in simple constructions the number of generations is divided by $|G|$, leading to models with realistically low numbers of generations [1]. Moreover, one can naturally solve the doublettriplet splitting problem $[12,13]$ in this setting.

${ }^{1}$ Closely related simultaneous work appears in Ref. [4]. 
More importantly, the nontrivial fundamental group allows us to introduce Wilson lines. In addition to being an attractive method of GUT symmetry breaking, Wilson lines are actually indispensable, as standard heterotic string models do not admit adjoint Higgs fields of the GUT group [12].

We will add the stabilization of moduli to this list of problems that admit natural solutions on Calabi-Yau manifolds with a nontrivial fundamental group and nontrivial gauge connection. The dilaton, Kähler moduli, and complex structure moduli can all be stabilized by incorporating the effects of gaugino condensation and the flux induced by the Wilson lines.

We would like to underscore the fortuitous coincidence that the necessary ingredients for our construction are automatically present in certain realistic models. Wilson lines typically lead to Chern-Simons flux, as we will explain in Sec. III C. Thus, heterotic string GUT models with Wilsonline symmetry breaking often have a background flux and an associated constant term in the superpotential. To the best of our knowledge the consequences of this term have not been well explored in the literature. In a restricted subset of models, namely those with hidden-sector gaugino condensation and very small Chern-Simons flux, the effect is dramatic: the moduli can be fixed, in a controllable regime, by the mechanism we are proposing.

The organization of this paper is as follows. In Sec. II we review basic facts about the relevant supergravity Lagrangians in ten and four dimensions and about the superpotential generated by gaugino condensation in the hidden $E_{8}$. In Sec. III we review the quantization conditions on three-form flux and describe how fractional flux can arise in the presence of flat connections with fractional ChernSimons invariant. In Sec. IV we describe how the fractional flux of Sec. III can be combined with gaugino condensation to stabilize the dilaton at weak coupling, along with the complex structure moduli. In Sec. V we include loop corrections and show that it becomes possible to simultaneously stabilize the Kähler moduli as well as the dilaton; this requires more restrictive assumptions about the choices of gauge bundles. We observe that a strong-coupling transition naturally arises in this setting, and we provide a toy model that illustrates the smoothness of this transition. In Sec. VI we discuss some basic aspects of the dual descriptions of our story, including the dual type IIA theories with wrapped D6-branes. In Sec. VII we explore the nature of the domain walls that interpolate between configurations with distinct fractional ChernSimons invariants. We conclude with a discussion of possible extensions and broader issues in Sec. VIII. Recently, three papers that have some overlap with our results appeared [14-16].

\section{GAUGINO CONDENSATION IN THE HETEROTIC STRING}

In this section we review the structure of the heterotic string low-energy effective Lagrangian, with particular attention to terms coupling the heterotic three-form flux, $H$, to the gauginos. In Sec. III A we fix notation by presenting the low-energy action for the heterotic string in ten dimensions.
We dimensionally reduce this action on a Calabi-Yau threefold space and describe the potential appearance of a gaugino condensate in the resulting $\mathcal{N}=1, d=4$ configuration. In Sec. II B we show how to derive the four-dimensional action of Sec. II A from a simple superpotential induced by the flux and the gaugino condensate. In Sec. II C we explain that the dilaton potential does not have a minimum at finite coupling unless the background flux is fractional.

\section{A. Effective Lagrangian for the heterotic theory}

The low-energy effective action for the heterotic string in ten-dimensional Einstein frame is [17]

$$
\begin{aligned}
S= & \frac{1}{2 \alpha^{\prime 4}} \int d^{10} x \sqrt{-g_{10}}\left[\mathcal{R}_{10}-\frac{1}{2} \partial_{A} \phi \partial^{A} \phi\right. \\
& -\frac{1}{12} e^{-\phi}\left(H_{A B C}-\frac{\alpha^{\prime}}{16} e^{\phi / 2} \bar{\chi}_{10} \Gamma_{A B C} \chi_{10}\right)^{2} \\
& \left.-\frac{\alpha^{\prime}}{4} e^{-\phi / 2} \operatorname{tr}\left(F_{A B} F^{A B}\right)-\alpha^{\prime} \operatorname{tr} \bar{\chi}_{10} \Gamma^{A} D_{A} \chi_{10}\right] .
\end{aligned}
$$

Indices $A, B$ run from 0 to 9 , and $\mu, \nu$ are four-dimensional spacetime indices. The internal space has real indices $m, n$ and (anti)holomorphic indices $i, j, \bar{i}, \bar{j}$. The Einstein-frame metric $g_{10}$ has Ricci scalar $\mathcal{R}_{10}$, while $\omega$ is the spin connection and $\phi$ is the dilaton. The heterotic string has gauge field strength $F_{\mu \nu}$ and gaugino field $\chi_{10}$; all traces are taken in the fundamental representation. The three-form flux $H_{A B C}$ is defined by

$$
H=d B-\frac{\alpha^{\prime}}{4}\left[\Omega_{3}(A)-\Omega_{3}(\omega)\right],
$$

where $\Omega_{3}$ is the Chern-Simons three-form,

$$
\Omega_{3}(A) \equiv \operatorname{tr}\left(A \wedge d A+\frac{2}{3} A \wedge A \wedge A\right)
$$

with a similar formula for $\Omega_{3}(\omega)$.

To reduce to four-dimensional Einstein frame, we use the ansatz

$$
d s_{10}^{2}=e^{-6 \sigma} d s_{4}^{2}+e^{2 \sigma} g_{m n}^{0} d y^{m} d y^{n},
$$

where $g_{m n}^{0}$ is a fixed fiducial metric normalized to have volume $4 \alpha^{\prime 3}$. Although this differs from the usual convention

$$
d s_{10}^{2}=e^{-6\left(\sigma-\sigma_{0}\right)} d s_{4}^{2}+e^{2 \sigma} g_{m n}^{0} d y^{m} d y^{n}
$$

by a constant rescaling, (2.4) is nevertheless appropriate for a discussion of moduli stabilization, as we do not know what the vacuum expectation value (VEV) $\sigma_{0}$ will be until we stabilize $\sigma$. For a similar reason, we go between tendimensional string and Einstein frame with the unconventional scaling $g_{M N}^{S}=g_{M N}^{E} e^{\phi / 2}$, while one usually sees $g_{M N}^{S}$ $=g_{M N}^{E} e^{\left(\phi-\phi_{0}\right) / 2}[18]$. The resulting Minkowski metric differs from the conventional $\operatorname{diag}(-1,1,1,1)$ by a constant scaling depending on the VEV's of the dilaton and volume modulus. To relate dimensionful quantities here to those directly mea- 
sured from experiments, one must perform an inverse rescaling. Finally, note that the $\Gamma$ matrices built from the metric scale with $e^{\sigma}$.

Let us decompose the ten-dimensional Majorana-Weyl gaugino $\chi_{10}$ as

$$
\chi_{10}=\chi_{6}^{*} \otimes \chi_{4}+\chi_{6} \otimes \chi_{4}^{*},
$$

where $\chi_{6}$ and $\chi_{4}$ are six and four-dimensional Weyl spinors with positive chirality and $\chi_{6}$ is the zero mode of the internal Dirac operator for the gaugino, with the normalization

$$
\chi_{6}^{\dagger} \chi_{6}=1
$$

We will choose to express the action in terms of a rescaled four-dimensional gaugino $\lambda$

$$
\lambda \equiv \chi_{4} e^{-9 \sigma / 2+\phi / 4}
$$

which will give the standard kinetic term after dimensional reduction.

\section{Coupling constants}

The four-dimensional gauge coupling is

$$
g_{\mathrm{YM}}^{2} \equiv e^{\varphi} \text {. }
$$

where the four-dimensional dilaton $\varphi$ is related to the tendimensional dilaton and volume modulus via

$$
\varphi=\phi / 2-6 \sigma
$$

Another important scalar field of the four-dimensional theory is the volume scalar ${ }^{2} \rho$,

$$
\rho=\phi / 2+2 \sigma
$$

The fields $\varphi$ and $\rho$ are related to the scalar components of two $\mathcal{N}=1$ chiral superfields $S$ and $T$ :

$$
\begin{aligned}
& S=e^{-\varphi}+i a, \\
& T=e^{\rho}+i b,
\end{aligned}
$$

where $a$ and $b$ are the axions that arise from the spacetime and internal components of $B_{A B}$, respectively. In particular,

$$
(* d a)_{\mu \nu \rho}=e^{-2 \varphi} H_{\mu \nu \rho}
$$

with an analogous relation for $b$.

The holomorphic Wilsonian gauge coupling functions $f_{i}^{W}$ (where $i=1,2$ runs over the two $E_{8}$ gauge groups) can be expressed in terms of $S$ and $T$ by

$$
f_{i}^{W}=S+\beta_{i} T+\mathcal{O}\left(e^{-S}\right)+\mathcal{O}\left(e^{-T}\right),
$$

${ }^{2}$ For the moment we assume that the Calabi-Yau manifold has only one volume modulus. We will present the more general case in Sec. V B. where the coefficient $\beta_{i}$ represents the one-loop correction to the gauge coupling function, and the last two terms represent nonperturbative corrections. Higher-loop corrections vanish by standard holomorphy arguments, since the dilaton and radion are partnered in chiral multiplets with axions. The physical effective coupling differs from the Wilsonian coupling by wave-function renormalization and integration over the low-momentum modes.

\section{Four-dimensional action}

Combining the relations given above, we reach the dimensionally reduced action ${ }^{3}$

$$
\begin{aligned}
S_{4 \mathrm{D}} & =S_{\text {gravity }}+S_{\text {gauge }}+S_{\mathrm{CY}}, \\
S_{\text {gravity }} & =\frac{2}{\alpha^{\prime}} \int d^{4} x \sqrt{-g_{4}}\left(\mathcal{R}_{4}-\frac{1}{2} \partial_{\mu} \varphi \partial^{\mu} \varphi-\frac{3}{2} \partial_{\mu} \rho \partial^{\mu} \rho\right),
\end{aligned}
$$

$$
\begin{aligned}
S_{\text {gauge }}= & \int d^{4} x \sqrt{-g_{4}}\left(-\frac{1}{2 g_{\mathrm{YM}}^{2}} \operatorname{tr}\left(F_{\mu \nu} F^{\mu \nu}\right)\right. \\
& \left.-\frac{2}{g_{\mathrm{YM}}^{2}} \operatorname{tr}\left(\bar{\lambda} \Gamma^{\mu} D_{\mu} \lambda\right)\right), \\
S_{\mathrm{CY}}= & -\frac{1}{24 \alpha^{\prime 4}} \int d^{4} x \sqrt{-g_{4}} e^{\varphi-3 \rho} \\
& \times \int_{X} d^{6} y \sqrt{-g^{0}}\left(H_{l m n}-\frac{\alpha^{\prime}}{16} e^{12 \sigma} T_{l m n}\right)^{2},
\end{aligned}
$$

where we have defined

$$
T_{l m n}=\operatorname{tr}\left[\left(\chi_{6}^{\dagger} \bar{\lambda}_{D}^{*}+\chi_{6}{ }^{T} \bar{\lambda}_{D}\right) \Gamma_{l m n}^{0}\left(\chi_{6}^{*} \lambda_{D}+\chi_{6} \lambda_{D}^{*}\right)\right],
$$

and $\lambda_{D}$ is the Dirac spinor corresponding to $\lambda$. The perfectsquare interaction term (2.18) couples the background flux to the gauginos and therefore gives rise, as we will see in detail, to a potential for the dilaton.

\section{Gaugino condensation}

Recall that in a pure $\mathcal{N}=1$ supersymmetric Yang-Mills theory in four dimensions with gauge group $H$, the gaugino condensate that develops at low energies is given by [19-22]:

$$
\begin{aligned}
\left\langle\operatorname{tr}\left[\frac{1}{2} \bar{\lambda}_{D}\left(1-\gamma_{5}\right) \lambda_{D}\right]\right\rangle & =\left\langle\operatorname{tr}\left(\lambda_{\alpha} \lambda^{\alpha}\right)\right\rangle \\
& =16 \pi^{2} M^{3} \exp \left(-\frac{8 \pi^{2} f^{W}}{C_{H}}\right) .
\end{aligned}
$$

\footnotetext{
${ }^{3}$ The unusual gravitational coupling $\kappa_{4}^{2}=\alpha^{\prime} / 4$ is an artifact of our ansatz (2.4). The physical gravitational coupling differs from this by the constant rescaling mentioned previously.
} 
Here $M$ is the ultraviolet cutoff for the gauge theory, $f^{W}$ is given by (2.14), and $C_{H}$ denotes the dual Coxeter number of $H$. We are interested in studying a gaugino condensate in some subgroup $H$ of the hidden-sector $E_{8}$ gauge group that arises in compactification of the $E_{8} \times E_{8}$ heterotic string on a Calabi-Yau manifold. The appropriate ultraviolet cutoff $M$ for a string compactification is the mass scale of KaluzaKlein excitations,

$$
M^{3}=c\left(\frac{e^{-12 \sigma}}{2 \alpha^{\prime 3 / 2}}\right),
$$

where $c$ is a constant of order one. Combining (2.20) and (2.21), we find that the gaugino condensate in $H \subset E_{8}$ satisfies

$$
\langle\operatorname{tr}(\lambda \lambda)\rangle=8 \pi^{2} c\left(\frac{e^{-12 \sigma}}{\alpha^{\prime 3 / 2}}\right) \exp \left(-\frac{8 \pi^{2} f^{W}}{C_{H}}\right) .
$$

\section{B. Superpotential from flux and a gaugino condensate}

For a variety of reasons it will prove useful to work with a superpotential and Kähler potential from which one can reproduce the interaction (2.18). One can derive the kinetic terms in (2.16) using the Kähler potential

$$
\mathcal{K}=-\log (S+\bar{S})-3 \log (T+\bar{T})-\log \left(-\frac{i}{4 \alpha^{\prime 3}} \int \Omega \wedge \bar{\Omega}\right) .
$$

The superpotential for this system takes the form

$$
W=W_{\text {flux }}+W_{\text {condensate }},
$$

where the first term is induced by the background flux and the second term is a nonperturbative contribution arising from the gaugino condensate.

The flux-induced superpotential can be written as an integral over the Calabi-Yau space [23-26]

$$
W_{\text {flux }}=\frac{2 \sqrt{2}}{\alpha^{\prime 4}} \int H \wedge \Omega .
$$

This superpotential leads to the following term in the scalar potential:

$$
V_{\text {flux }}=\frac{1}{24 \alpha^{\prime 4}} e^{\varphi-3 \rho} \int_{X} d^{6} y \sqrt{-g^{0}} H_{l m n} H^{l m n},
$$

which is precisely the first term in (2.18). As we will explain in Sec. III, the number of quanta of $H$ flux is roughly given by

$$
h=\frac{1}{4 \pi^{2} \alpha^{\prime 4}} \int H \wedge \Omega
$$

so that we may define a mass parameter $\mu$,

$$
\mu^{3}=\frac{4 \sqrt{2} c \pi^{2}}{\alpha^{\prime 3 / 2}}
$$

in terms of which

$$
W_{\text {flux }}=\left(\frac{2 \mu^{3}}{c}\right) h .
$$

The nonperturbative contribution is conveniently expressed in terms of the Wilsonian coupling [27]

$$
W_{\text {condensate }}=-C_{H} \mu^{3} \exp \left(-\frac{8 \pi^{2} f^{W}}{C_{H}}\right),
$$

where the normalization was obtained by comparing to (2.18). Putting these two pieces together, the total superpotential is

$$
W=\left(\frac{2 \mu^{3}}{c}\right) h-C_{H} \mu^{3} \exp \left(-\frac{8 \pi^{2} f^{W}}{C_{H}}\right) .
$$

\section{Conditions for a stabilized dilaton}

A potential for the dilaton arises from the perfect-square interaction term (2.18), which couples the background flux to the gauginos. To analyze this expression we first observe that the gaugino bilinear appearing in (2.18) is proportional to the covariantly constant holomorphic three-form. This follows from the fact that $\chi_{6}$ is a gaugino zero mode on the CalabiYau manifold [3]:

$\operatorname{tr}\left[\left(\chi_{6}{ }^{\dagger} \bar{\chi}^{*}+\chi_{6}{ }^{T} \bar{\chi}\right) \Gamma_{l m n}^{0}\left(\chi_{6}^{*}+\chi_{6} \chi^{*}\right)\right]=2\langle\operatorname{tr}(\lambda \lambda)\rangle \Omega_{l m n}+$ c.c.

Here $\Omega$ is the holomorphic $(3,0)$ form on the Calabi-Yau manifold, with the normalization (1/3!) $\Omega_{i j k} \bar{\Omega}^{i j k}=1$.

Minimizing the perfect square (2.18) forces $\langle\lambda \lambda\rangle \Omega$ $+\langle\lambda \lambda\rangle^{*} \bar{\Omega}$ to align itself along the same direction in $H^{3}(M, R)$ as the three-form flux $H$. This uniquely fixes the complex structure moduli and the four-dimensional gaugino condensate. Because the gaugino condensate depends on the four-dimensional dilaton, it follows that the interaction (2.18) generates a potential for the dilaton.

However, the minimum of this potential is generically at infinite coupling. In the absence of Chern-Simons contributions, the three-form $H$ obeys the quantization condition

$$
\frac{1}{2 \pi^{2} \alpha^{\prime}} \int_{Q} d B=n
$$

for any $Q$ in $H_{3}(X, Z)$. The second term inside the perfect square of (2.18), on the other hand, integrates over threecycles to

$$
\begin{aligned}
& \int_{Q} \frac{\alpha^{\prime} e^{12 \sigma}}{8}\left[\langle\operatorname{tr}(\lambda \lambda)\rangle \Omega_{i j k}+\text { c.c. }\right] \\
& \quad=\frac{c \pi^{2}}{\alpha^{\prime 1 / 2}} \exp \left(-\frac{8 \pi^{2}}{C_{H} g_{\mathrm{YM}}^{2}}\right)\left(e^{-i \theta} \int_{Q} \Omega+e^{+i \theta} \int_{Q} \bar{\Omega}\right) \\
& \simeq c \pi^{2} \alpha^{\prime} \exp \left(-\frac{8 \pi^{2}}{C_{H} g_{\mathrm{YM}}^{2}}\right) .
\end{aligned}
$$


These two terms cancel only if

$$
\frac{c}{2} \exp \left(-\frac{8 \pi^{2}}{C_{H} g_{\mathrm{YM}}^{2}}\right) \simeq n \text {. }
$$

This has no solution because the left-hand side is almost always ${ }^{4}$ less than one. This means that instead of stabilizing the four-dimensional dilaton at a finite value, turning on an integral flux $d B$ actually drives the system to infinitely strong coupling. Our proposal is to use fractional fluxes to overcome this problem and stabilize $g_{\mathrm{YM}}$ at finite coupling. We therefore turn to an investigation of the conditions under which fractional flux can arise in the heterotic string.

\section{FRACTIONAL FLUX INDUCED BY GAUGE FIELDS}

In Sec. III A we review the quantization condition for three-form flux and explain its relation to the Chern-Simons invariant. In Sec. III B we briefly discuss the class of threemanifolds used in our models and construct a simple example. In Sec. III C we provide expressions for the ChernSimons invariants of these manifolds. In Sec. III D we discuss the conditions under which the fractional ChernSimons flux leads to a worldsheet anomaly, and we explain how this can be avoided in our setup.

\section{A. Quantization conditions for three-form flux}

Consider a compactification of the $E_{8} \times E_{8}$ heterotic string on a Calabi-Yau manifold $X$. The two-form $B_{\mu \nu}$ is required to satisfy

$$
\frac{1}{2 \pi^{2} \alpha^{\prime}} \int_{Q} d B=n
$$

for any three-cycle $Q$ in $H_{3}(X, Z)$ in order for the action of worldsheet instantons to be single-valued [5]. However, the gauge-invariant field strength is

$$
H=d B-\frac{\alpha^{\prime}}{4} \Omega_{3}(A)+\frac{\alpha^{\prime}}{4} \Omega_{3}(\omega) .
$$

This does not need to obey the same quantization law, due to the presence of the Chern-Simons term. To see this let us assume for simplicity that the background $B$ field is trivial, and that the contribution of the spin connection $\omega$ can be ignored. Then only the remaining factor of the gauge connection contributes. So instead of (3.1) we find the quantization rule

$$
\frac{1}{2 \pi^{2} \alpha^{\prime}} \int_{Q} H=-C S(A, \dot{Q}),
$$

where we introduced a standard notation

\footnotetext{
${ }^{4} \mathrm{We}$ are assuming that the constant $c$ in (2.21) is of order one. If $c$ takes a larger value in a particular model, then integral flux might possibly stabilize the dilaton, albeit at relatively strong coupling. We will not investigate this possibility here.
}

$$
C S(A, Q)=\frac{1}{8 \pi^{2}} \int_{Q} \Omega_{3}(A)=\frac{1}{8 \pi^{2}} \int_{Q} \operatorname{tr}\left(A \wedge d A+\frac{2}{3} A \wedge A \wedge A\right)
$$

for the Chern-Simons invariant associated with a threemanifold $Q$ and a connection one-form $A$.

The invariant $C S(A, Q)$ plays an important role in the theory of three-manifolds. In particular, if $V^{\prime}$ is a gauge bundle over $Q$ and if $A$ is a flat gauge connection on $V^{\prime}$, then $C S(A, Q)$ is a topological invariant, in the sense that $C S(A, Q)$ takes a fixed value on each component of the moduli space of flat connections on $Q$. Moreover, it is well known that $C S(A, Q)$ is well defined only modulo integers and can take fractional values. If we further assume that the bundle $V^{\prime}$ pulls back to a gauge bundle $V$ over the CalabiYau manifold $X$, then we obtain the desired situation where the three-form flux takes fractional values. In the following sections we will use this as a mechanism to produce small quanta of the $H$ flux, which can then be used to stabilize the various moduli.

\section{B. Three-cycles with fractional flux}

Certain classes of three-cycles in Calabi-Yau manifolds admit connections with fractional Chern-Simons invariants. We now turn to a discussion of the properties of such threecycles.

Since only holomorphic and antiholomorphic components of the three-form flux contribute to the superpotential (2.25), the only fractional fluxes we need to consider are those of Hodge type $(3,0)+(0,3)$. These can be viewed as fluxes through special Lagrangian cycles $Q$. Typically these are compact three-manifolds with non-negative curvature that support gauge fields suitable for our purposes. According to McLean [28], the deformations of a special Lagrangian submanifold $Q$ can be identified with the harmonic one-forms on $Q$. Specifically, the deformation space has real dimension $b_{1}(Q)$. Therefore, rigid special Lagrangian three-cycles are precisely rational homology three-spheres, i.e., threemanifolds with $b_{1}(Q)=0$. We shall henceforth restrict our attention to rigid special Lagrangian three-cycles. The local Calabi-Yau geometry near such cycles is always of the form

$$
T^{*} Q
$$

For example, we can choose $Q$ to be the base of the special Lagrangian torus fibration [29],

$$
f: X \rightarrow Q
$$

Indeed, following Strominger, Yau, and Zaslow [29], consider a Bogomol'nyi-Prasad-Sommerfield (BPS) state in the effective four-dimensional theory represented by $N$ D6branes wrapped over the entire mirror manifold $\tilde{X}$. These D6-branes are rigid and, because the fundamental group of $\widetilde{X}$ is finite, there is only a discrete set of Wilson lines. In fact, the latter account for the degeneracy of D-brane bound states [30]. Namely, the number of bound states of $N$ D-branes is given by the number of $N$-dimensional irreducible represen- 
tations of $\pi_{1}(\tilde{X})$. Under mirror symmetry (realized as $T$ duality on $T^{3}$ fibers) these D6-branes become D3-branes wrapped around the base $Q$. In order for the D3-branes to have no continuous moduli the base manifold $Q$ must be a rational homology three-sphere. Also, by looking at the degeneracy of D-brane bound states for different values of $N$, we conclude that $\pi_{1}(Q)$ and $\pi_{1}(\widetilde{X})$ should be related. Notice that since both $X$ and its mirror $\tilde{X}$ are fibered over the same base $Q$, the above arguments imply that their homotopy groups should be related as well. In particular, in a large class of examples one finds that the Abelian parts of $\pi_{1}(X)$ and $\pi_{1}(\tilde{X})$ are isomorphic; cf. Ref. [31].

Let us study a simple example that will be relevant in the following. Consider a quintic hypersurface in $\mathbf{C P}^{4}$,

$$
z_{1}^{5}+z_{2}^{5}+z_{3}^{5}+z_{4}^{5}+z_{5}^{5}+(\text { deformations })=0 .
$$

This hypersurface represents a Calabi-Yau variety $X_{0}$ with $h^{1,1}=1, h^{2,1}=101$. Unfortunately, $\pi_{1}\left(X_{0}\right)$ is trivial, so $X_{0}$ does not admit a fractional flux induced by nontrivial gauge fields. Moreover, since the number of generations in a heterotic compactification on a Calabi-Yau threefold $X$ is related, in the case of the standard embedding, to the Euler number of $X[1]$, in the present case with the standard embedding we find an unrealistically large number, $N$ $=\frac{1}{2}\left|\chi\left(X_{0}\right)\right|=100$. A model with a more realistic spectrum that does not suffer from these problems can be obtained by considering a quotient of $X_{0}$,

$$
X=X_{0} / \Gamma
$$

by a discrete symmetry group $\Gamma=Z_{5} \times Z_{5}$, generated by two elements

$$
\begin{aligned}
& g_{1}:\left(z_{1}, z_{2}, z_{3}, z_{4}, z_{5}\right) \rightarrow\left(z_{5}, z_{1}, z_{2}, z_{3}, z_{4}\right), \\
& g_{2}:\left(z_{1}, z_{2}, z_{3}, z_{4}, z_{5}\right) \rightarrow\left(\zeta z_{1}, \zeta^{2} z_{2}, \zeta^{3} z_{3}, \zeta^{4} z_{4}, z_{5}\right),
\end{aligned}
$$

where $\zeta=\exp (2 \pi i / 5)$. Since $\Gamma$ acts freely on $X_{0}$, we have $\chi(X)=\chi\left(X_{0}\right) / 25=8$ and $\pi_{1}(X)=Z_{5} \times Z_{5}$. Therefore, compactification of the heterotic string on the resulting manifold $X$ with the standard embedding provides a model with only four generations, and there is a possibility to turn on nontrivial Wilson lines on $X$. Also, it is easy to see that the base $Q$ of the special Lagrangian torus fibration in this case is a rational homology three-sphere with nontrivial fundamental group.

For the quintic hypersurface (3.6), the base $Q_{0}$ of the special Lagrangian torus fibration can be represented by the image of the moment map, $z_{i} \rightarrow\left|z_{i}\right|^{2}$. The topology of $Q_{0}$ can easily be understood in the large complex structure limit, where it is close to the boundary of the toric polytope. Hence, $Q_{0} \cong \mathbf{S}^{3}$. Now let us consider the action of the discrete group $\Gamma$. From (3.7) it follows that the generator $g_{2}$ acts trivially on $Q_{0}$, whereas $g_{1}$ acts freely. Therefore, we find that the base of the special Lagrangian torus fibration $X$ $\rightarrow Q$ is a Lens space,

$$
Q=\mathbf{S}^{3} / Z_{5}
$$

In particular, we have $\pi_{1}(Q)=Z_{5}$ and, as we will show below, there are many choices for the gauge bundle $V^{\prime}$ and for the gauge connection $A$ over this three-manifold, such that $C S(A, Q)$ has fractional values. If $V^{\prime}$ is such a bundle, we can define its pullback $V=f^{-1} V^{\prime}$ under the projection map (3.5). The resulting gauge bundle $V$ over $X$ has the desired properties and, according to the quantization rule (3.3), the three-form flux in heterotic string theory on this background can take fractional values.

This construction can easily be generalized to an arbitrary special Lagrangian three-cycle $Q$ that is rigid inside $X$. As was explained above, the condition of rigidity implies that $Q$ is a rational homology three-sphere. Examples of rational homology three-spheres that can occur as special Lagrangian cycles in Calabi-Yau threefolds include Lens spaces, Brieskorn homology three-spheres, and, more generally, Seifert fibered three-manifolds. Recall that the Seifert threemanifold, $\Sigma\left(a_{1}, \ldots, a_{n}\right)$, is a circle fibration over a twosphere, with $n$ multiple fibers. This includes Brieskorn spheres and Lens spaces as a special case, $n=3$. For instance, the Lens space $L(p, 1)=\mathbf{S}^{3} / \mathbb{Z}_{p}$ is a Seifert threemanifold with $\left(a_{1}, a_{2}, a_{3}\right)=(p, 2,2)$. Many of these threemanifolds support nontrivial gauge connections with fractional Chern-Simons functional [32,33].

\section{Formulas for the Chern-Simons invariant}

In order to determine the set of values of $C S(A, Q)$ for a given three-manifold $Q$, one has to study the space of representations of the fundamental group, $\pi_{1}(Q)$, into the gauge group. A familiar example of a reducible ${ }^{5}$ gauge connection on a manifold with $\pi_{1}=\mathbb{Z}_{p}$ corresponds to a discrete Wilson line of the form

$$
U=\operatorname{diag}\left(e^{2 \pi i k_{1} / p}, \ldots, e^{2 \pi i k_{8} / p}\right)
$$

variations of which are often used to break the GUT gauge group to a smaller subgroup, such as the Standard Model gauge group [12]. The Chern-Simons invariant of such a connection is [35] (see also Ref. [36])

$$
C S(A, Q)=\sum_{i} \frac{k_{i}^{2}}{2 p} \quad \bmod \mathbb{Z}
$$

where the sum is over all eight complex worldsheet fermions. For appropriate choices of $p$ and of the $k_{i}$ the result is a

\footnotetext{
${ }^{5} \mathrm{~A}$ connection $A$ is called reducible if its isotropy subgroup, that is, a maximal subgroup that commutes with all the holonomies of $A$, is a continuous group. Otherwise, $A$ is called irreducible. For example, an $\mathrm{SU}(2)$ gauge connection is reducible if its isotropy subgroup is $\mathrm{U}(1)$. Notice that reducible gauge connections may have nonzero Chern-Simons invariant; see, e.g., Ref. [34].
} 
fractional Chern-Simons invariant. ${ }^{6}$ This has the surprising consequence mentioned in the Introduction: in many cases the Wilson lines that are used to break the GUT gauge group to the standard model introduce a fractional Chern-Simons invariant, and hence a fractional flux.

We now turn to the more general question of the fractional Chern-Simons invariants of Seifert three-manifolds; this choice covers a fairly large class of models relevant to the physical problem at hand. Without loss of generality, we can take the gauge group to be $\mathrm{SU}(2)$ (which can be realized as a subgroup in one of the two $E_{8}$ 's). Let $Q$ $=\Sigma\left(a_{1}, \ldots, a_{n}\right)$ be a Seifert three-manifold. In this case, the irreducible representations

$$
\rho: \quad \pi_{1}(Q) \rightarrow \mathrm{SU}(2)
$$

are characterized by what are called "rotation numbers" $\left( \pm m_{1}, \ldots, \pm m_{n}\right)$, where each $m_{i}$ is defined modulo $a_{i}$,

$$
m_{i} \sim m_{i}+a_{i} .
$$

Furthermore, there exists at most one component of the representation variety realizing a given set of rotation numbers $\left(m_{1}, \ldots, m_{n}\right)$. If $A$ is the corresponding connection oneform, the value of the Chern-Simons functional, $C S(A, Q)$, is given by the simple formula

$$
C S(A, Q)=-\sum_{i=1}^{3} \frac{1}{a_{i}}\left(m_{i}+\lambda\right)^{2},
$$

where

$$
\lambda=0, \frac{1}{2} \text {. }
$$

In particular, if $Q=\mathbf{S}^{3} / Z_{p}$ is a Lens space, from the general formula (3.11) we find

$$
C S(A, Q)=-\frac{1}{p}\left(m_{1}+\lambda\right)^{2}-\frac{\lambda^{2}}{2} \bmod \mathbb{Z},
$$

where for simplicity we set $m_{2}=m_{3}=0$. This expression gives two sets of values of the Chern-Simons functional (listed in Ref. [32]) corresponding to $\lambda=0$ and $\lambda=\frac{1}{2}$, respectively. It is convenient to introduce a new integer parameter

$$
m=2 m_{1}+2 \lambda \quad \bmod 2 p
$$

and rewrite (3.13) in the form

$$
C S(A, Q)=-\frac{m^{2}}{4 p}-\frac{\lambda^{2}}{2} \bmod \mathbb{Z}
$$

In general, it follows from (3.11) that $C S(A, Q)$ is a rational number whose denominator can be as large as the order of the fundamental group, $\pi_{1}(Q)$.

\footnotetext{
${ }^{6}$ In Sec. III D we review the existence and cancellation of a potential worldsheet global anomaly in such backgrounds.
}

\section{A global worldsheet anomaly from fractional Chern-Simons invariants}

For completeness, we now discuss a technical issue related to modular invariance in a fractional flux background. Specifically, we present a sufficient condition for cancellation of the worldsheet anomaly induced by fractional ChernSimons flux. ${ }^{7}$

When the heterotic string propagates on a nontrivial geometry $M$ with nontrivial Wilson lines, there is a global worldsheet anomaly in addition to the one-loop anomaly seen in the ten-dimensional supergravity [35]. This signals that the worldsheet instanton path integral is not necessarily single-valued in such a background.

To compute the anomaly, consider a one-parameter $(t)$ family of maps from a one-parameter family of worldsheets into the target space, with the worldsheets at $t=0$ and $t=1$ identified by a large diffeomorphism $h$ preserving the spin structure $\varphi:\left(\Sigma \times[0,1]_{t}\right)_{h} \rightarrow M$. The change of the fermion determinant can be calculated using an index theorem [35],

$$
\ln Z\left(\phi^{i}, t=1\right)-\ln Z\left(\phi^{i}, t=0\right)=-2 \pi i \int_{\varphi(\Sigma \times[0,1])_{h}} \Omega_{3}(A),
$$

where

$$
Z\left(\phi^{i}, t ; g_{i j}, B_{i j}, A_{i B}^{A}\right)=\left(\operatorname{det}_{T}^{+}\right)\left(\operatorname{det}_{V_{1}}^{-}\right)\left(\operatorname{det}_{V_{2}}^{-}\right)\left(\operatorname{det}^{+} R\right) .
$$

Here the first three terms inside the logarithm are Dirac determinants for the right- and left-moving fermions coupled to the pullback of the spin connection and gauge connection, and the fourth term comes from the right-moving RaritaSchwinger ghost. If we were unable to find other sources to cancel the factor on the right-hand side, we would have to set the Chern-Simons invariant to an integer to maintain the single-valuedness of the determinants.

Fortunately the Wess-Zumino term on the worldsheet can help us. For the heterotic string on a Calabi-Yau manifold with flat $B$ field and with no Wilson lines, the worldsheet action looks like

$$
\begin{aligned}
S= & \int d^{2} x\left\{\left[g_{i j}(\phi)+B_{i j}(\phi)\right] \partial_{+} \phi^{i} \partial_{-} \phi^{j}+i g_{i j} \psi^{i}\left(\partial_{-} \psi^{j}\right.\right. \\
& \left.+\Gamma_{j k}^{i} \partial_{-} \phi^{k} \psi^{l}\right)+i G_{A B}(\phi) \lambda^{A}\left[\partial_{+} \lambda^{B}+A_{i}{ }_{B}{ }_{B}\left(\partial_{+} \phi^{i}\right) \lambda^{C}\right] \\
& \left.+\frac{1}{2} \widetilde{F}_{i j A B} \psi^{i} \psi^{j} \lambda^{A} \lambda^{B}\right\},
\end{aligned}
$$

where $\psi^{i}$ and $\lambda^{A}$ are the right- and left-moving fermions, $\Gamma_{j k}^{i}$ is the Levi-Civita connection of the target space, and $G_{A B}$ is the metric on the gauge bundle. This action has manifest $(0,2)$ supersymmetry. The question is, if we now turn on flat Wilson lines supporting the fractional Chern-Simons invariant, resulting in multivalued fermion determinants, can we

\footnotetext{
${ }^{7}$ We are indebted to E. Witten for explaining to us much of the content in this subsection.
} 
find canceling effects from the bosonic worldsheet action? The answer is yes, provided there is no torsion in $H^{4}(M, Z)$.

To see this, consider the following exact sequence:

$$
\begin{aligned}
\cdots & \rightarrow H^{3}(M, \mathbf{R}) \stackrel{e}{\rightarrow} H^{3}(M, U(1)) \\
& \stackrel{d}{\rightarrow} H^{4}(M, \mathbb{Z}) \rightarrow H^{4}(M, \mathbf{R}) \rightarrow \cdots .
\end{aligned}
$$

The Chern-Simons invariant $\exp \left[i \int \Omega_{3}(A)\right]$ for a flat bundle takes values in $H^{3}(M, U(1))$ and is mapped into the torsion part of $H^{4}(M, Z)$. If $H^{4}(M, Z)$ is torsion-free, the ChernSimons invariant lives in the kernel of $d$ and therefore $\Omega_{3}(A)$ lives in $H^{3}(M, \mathbf{R})$. So there exists, locally, a twoform $\widetilde{B}$ :

$$
d \widetilde{B}=\Omega_{3}(A) .
$$

It is crucial that $\widetilde{B}$ is not globally defined when $\int \Omega_{3}(A)$ is fractional. The change in phase from the coupling of $\widetilde{B}$ to the worldsheets cancels the change in the fermion determinants in Eq. (3.15) [37]. On the other hand, if $H^{4}(M, Z)$ has a torsion piece, $\widetilde{B}$ does not exist for bundles supporting fractional Chern-Simons invariant and we cannot cancel the global worldsheet anomaly. The only consistent Wilson lines are then those that give integer Chern-Simons fluxes.

The reader will have noticed that if we modify the WessZumino term into

$$
\int_{\Sigma} B+\widetilde{B}
$$

we no longer have $(0,2)$ worldsheet supersymmetry. We can preserve $(0,1)$ supersymmetry by modifying the connection to

$$
\widetilde{\Gamma}_{j k}^{i}=\Gamma_{j k}^{i}+g^{i l}(d \widetilde{B})_{j k l}=\Gamma_{j k}^{i}+g^{i l} \Omega(A)_{j k l} .
$$

However, the complex structure $J^{i}{ }_{j}$ is no longer covariantly constant. Thus, just as we expected, turning on a flat bundle with Chern-Simons gauge flux generates a spacetime superpotential $W=\int \Omega_{3}(A) \wedge \Omega$ and breaks $\mathcal{N}=1$ spacetime supersymmetry and $(0,2)$ worldsheet supersymmetry. It is obvious from the supergravity effective action that with the addition of a gaugino condensate, spacetime supersymmetry can be restored. However, we do not expect a useful worldsheet description after including such spacetime effects. ${ }^{8}$

\footnotetext{
${ }^{8}$ Alternatively, to preserve $(0,2)$ worldsheet supersymmetry, one could modify $J^{i}{ }_{j}$ so that $\tilde{\nabla}_{i} J^{j}{ }_{k}=J^{j}{ }_{k, i}+\widetilde{\Gamma}_{i l}{ }_{i} J^{l}{ }_{k}-\widetilde{\Gamma}_{i k}^{l} J^{j}{ }_{l}=0$ with respect to the modified connection. This typically cannot be achieved by a local modification (i.e., a continuous deformation) and requires starting with a non-Kähler manifold. This is closely related to Ref. [38] and to more recent literature on non-Kähler compactifications. The difference is that here we would consider non-Kähler behavior due to $\Omega_{3}(A)$ instead of the more conventional nonflat $d B$.
}

We have seen, then, that a sufficient condition for cancellation of the worldsheet anomaly in the presence of fractional flux is absence of torsion in $H^{4}(M, Z)$. More specifically, it is enough that no three-cycle $Q$ on which the Chern-Simons form integrates to a fraction is a torsion cycle in $H_{3}(M, Z)$. We will henceforth assume that this condition is satisfied.

\section{DILATON STABILIZATION}

We will now demonstrate that the combination of a gaugino condensate and a fractional flux induced by the Chern-Simons term of the $E_{8} \times E_{8}$ gauge connection can lead to stabilization of the dilaton at finite (and, with sufficient tuning, weak) coupling.

We denote the two gauge groups $E_{8}^{(i)}, i=1,2$. Let us henceforth adopt the convention that $E_{8}^{(1)}$ is the observable $E_{8}$ and $E_{8}^{(2)}$ is the hidden sector. We imagine that there is a suitable visible-sector bundle that breaks $E_{8}^{(1)}$ to an attractive GUT group. If a realistic model is desired, we may also require that the observable $E_{8}^{(1)}$ has a gauge bundle with $\left|\int c_{3}\right|=6$ to give three generations of quarks and leptons. ${ }^{9}$ In the remaining visible-sector group we then turn on Wilson lines which have fractional Chern-Simons invariant on some three-cycle. The resulting fractional flux generates a superpotential via $(2.25) .^{10}$

For the purposes of this section we could take the hiddensector bundle to be trivial, so that $E_{8}^{(2)}$ is unbroken. However, it will prove useful in Sec. V to include a nontrivial gauge bundle in each of the $E_{8}$ 's. We therefore embed an SU(2) bundle into $E_{8}^{(2)}$, breaking $E_{8} \rightarrow E_{7}$. There is no index theorem protecting charged matter in $E_{7}$ (as it has only real representations), so we can safely assume that the low-energy $E_{7}$ gauge theory in the hidden sector has no light fields transforming in the 56. The gauge group then confines at low energies, providing a gaugino condensate to balance the fractional flux, as in Sec. II B.

The overall result is the superpotential (2.31):

$$
\frac{W}{\mu^{3}}=\frac{2 h}{c}-18 \exp \left(-\frac{8 \pi^{2} S}{18}\right),
$$

where $h=\left(2 \pi^{2} \alpha^{\prime 5 / 2}\right)^{-1} \int H \wedge \Omega$ is the flux contribution and the second term is the result of gaugino condensation (the dual Coxeter number of $E_{7}$ is 18$) .{ }^{11}$

To look for a supersymmetric vacuum, we solve the equation $D_{S} W=0$, with the result

$$
h=\left[9 c+8 c \pi^{2} \operatorname{Re}(S)\right] \exp \left(-\frac{8 \pi^{2} S}{18}\right) .
$$

\footnotetext{
${ }^{9}$ Examples of Calabi-Yau models with three generations and nontrivial $\pi_{1}$ have appeared in Ref. [39], and undoubtedly many more could be constructed in a systematic search.

${ }^{10}$ The fractional flux could instead come from hidden-sector Wilson lines. We focus on visible-sector Wilson lines for simplicity.

${ }^{11}$ This superpotential is of the same form as the one appearing in, for instance, Eq. (12) of Ref. [40]. There, the small constant term comes from the $(0,3)$ part of the type IIB $G_{3}$ flux, while the exponential arises from nonperturbative gauge dynamics as in our system.
} 
Modest values of the Chern-Simons invariant lead to a solution at weak coupling. For example, if $h$ is approximately $\frac{1}{10}$, which is easily attainable using the constructions of Sec. III, then (4.2) can be solved with $\operatorname{Re}(S) \sim 1.6$, which corresponds to $\alpha_{\mathrm{GUT}} \sim \frac{1}{20}$. To achieve instead the often-quoted value $\alpha_{\text {GUT }} \sim \frac{1}{25}$, one needs $h$ of order $\frac{1}{40}$. Of course the requirements are weaker if we take the pure hidden-sector gauge group to be $E_{8}$ instead of $E_{7}$.

There are many variations of this mechanism that involve slightly different choices of bundles. It seems to us that the most elegant models are those in which one set of Wilson lines breaks the observable-sector GUT group to the Standard Model and also provides the needed fractional ChernSimons invariant.

We have already solved the dilaton equation $D_{S} W=0$. We can likewise solve the equations for the complex structure moduli by making $H$ of type $(3,0)+(0,3)$. In this way the $H$ flux from the Chern-Simons invariant generically stabilizes all complex structure moduli. The Kähler moduli of the Calabi-Yau manifold, however, are not yet fixed. In particular, there is a flat direction for the volume modulus $T .{ }^{12}$

In fact, this flat direction is a general property of "noscale" models. From the form (2.23) of the Kähler potential, combined with the fact that $W$ is independent of the volume modulus $T$ at this order, we see that the supergravity potential undergoes a simplification

$$
V=e^{K}\left(g^{i \bar{j}} D_{i} W \overline{D_{j} W}-3|W|^{2}\right) \rightarrow e^{K}\left(g^{a \bar{b}} D_{a} W \overline{D_{b} W}\right),
$$

where $i, j$ run over all fields, but $a, b$ run over all fields except $T$. As a result, we are left with a flat direction, $T$. Generically $D_{T} W \neq 0$, so supersymmetry is broken. Nevertheless, the vacuum energy vanishes at this order of approximation, since we have solved $D_{a} W=0$ for all $a$. Loop corrections will plausibly destabilize $T$, resulting in a runaway problem for the overall volume.

We will suggest a solution to this problem, in the context of Calabi-Yau compactification, in the next section. However, we should point out that investigation of supersymmetric non-Kähler compactifications of string theory has recently been renewed (see, e.g., Refs. $[41,42,15,43])$. In such compactifications the overall volume modulus can be stabilized at tree level by balancing fluxes against the non-Kähler nature of the geometry. The combination of this tree-level $T$ stabilization with our results on dilaton stabilization could plausibly yield weakly coupled models with all moduli stabilized. This would require a compactification manifold that admits moderately small Chern-Simons invariants.

\section{DILATON AND VOLUME STABILIZATION IN CALABI-YAU MODELS}

In Sec. V A we show that it is possible, with appropriate choices of bundles, to stabilize both the dilaton and the over-

\footnotetext{
${ }^{12}$ If there are vector bundle moduli then these are also unfixed. However, in Sec. VIII we explain why bundle moduli could be absent in generic situations.
}

all volume by incorporating the one-loop correction to the gauge coupling. In Sec. V B we extend this mechanism to stabilize all the Kähler moduli of a threefold. In Sec. V C we investigate the strong-coupling transition that occurs in these models. We present a toy model to illustrate the physical smoothness of this transition. In Sec. VD we discuss the conditions under which the resulting theory is weakly coupled. In Sec. VE we summarize our assumptions concerning the Calabi-Yau and the $E_{8}$ gauge bundles.

\section{A. One-loop correction}

We first consider, for simplicity, the case of a Calabi-Yau threefold that has $h^{1,1}=1$ and hence a single Kähler modulus. When one-loop corrections are incorporated, the Wilsonian gauge kinetic functions have the form (2.14):

$$
f_{(i)}^{W}=S+\beta_{i} T
$$

where $i=1,2$ labels the gauge groups $E_{8}^{(1)}, E_{8}^{(2)}$. In the case without space-filling heterotic five-branes, it is a simple matter to derive the linear terms in $T$ by dimensional reduction of the $B \wedge X_{8}\left(F_{1}, F_{2}, R\right)$ term in the ten-dimensional $E_{8}$ $\times E_{8}$ theory. The result is

$$
\begin{aligned}
& \beta_{1}=\frac{1}{8 \pi^{2}} \int_{X} J \wedge\left[c_{2}\left(V_{1}\right)-c_{2}\left(V_{2}\right)\right], \\
& \beta_{2}=\frac{1}{8 \pi^{2}} \int_{X} J \wedge\left[c_{2}\left(V_{2}\right)-c_{2}\left(V_{1}\right)\right] .
\end{aligned}
$$

Here $J$ is the generator of $H^{1,1}(X, Z)$. Notice that

$$
\beta_{1}+\beta_{2}=0
$$

while in the case of the standard embedding

$$
\beta_{1}-\beta_{2}=\frac{1}{4 \pi^{2}} \int_{X} J \wedge c_{2}(T X)
$$

This fact that the difference of the gauge coupling functions is given by a topological invariant (in the case of the standard embedding) was observed in, e.g., Ref. [44]. One can easily calculate $\beta$ for a few simple examples. We present the calculation below for $J \wedge c_{2}(T X)$; one can imagine partitioning this into $c_{2}\left(V_{1,2}\right)$ in various ways:

$$
\begin{gathered}
\int_{[4 \| 5]} J \wedge c_{2}=10 \int_{[4 \| 5]} J \wedge J \wedge J=50, \\
\int_{\left[\begin{array}{ll}
5 \| 3 & 3
\end{array}\right]} J \wedge c_{2}=6 \int_{\left[\begin{array}{lll}
5 \| 3 & 3
\end{array}\right]} J \wedge J \wedge J=54, \\
\int_{[6 \| 3 \quad 2 \quad 2]} J \wedge c_{2}=5 \int_{\left[\begin{array}{lll}
6 \| 3 & 2 & 2
\end{array}\right]} J \wedge J \wedge J=60 .
\end{gathered}
$$

From these examples it is plausible that $\beta$ can be reasonably large, at least of order one.

We will choose the gauge bundle $V_{2}$ so that $E_{8}^{(2)}$ is broken to a subgroup $H$ (say $E_{7}$ ) without any light charged matter. 
The resulting four-dimensional theory therefore has a sector that is pure $\mathcal{N}=1$ supersymmetric gauge theory with gauge group $H$, which undergoes gaugino condensation at low energies. Let us furthermore choose the bundle $V_{1}$ so that $E_{8}^{(1)}$ is broken to a low-energy group and matter content that can contain the Standard Model. Finally, we take $\beta_{2}=-\beta_{1}=\beta$ $>0$, so that $E_{8}^{(1)}$ is more strongly coupled than $E_{8}^{(2)} \cdot{ }^{13}$

The complete superpotential is then

$$
\frac{W}{\mu^{3}}=\frac{2 h}{c}-C_{H} \exp \left(-\frac{8 \pi^{2}}{C_{H}}(S+\beta T)\right) .
$$

This superpotential depends nontrivially on both of the chiral multiplets $S$ and $T$. The condition for a supersymmetric vacuum is

$$
W_{; S}=W_{; T}=0,
$$

where the Kähler covariant derivatives are determined using (2.23).

A solution of (5.7) necessarily satisfies

$$
\begin{aligned}
3 S & =\beta T, \\
h & =\left(\frac{C_{H} c}{2}+8 c \pi^{2} \operatorname{Re}(S)\right) \exp \left(-\frac{32 \pi^{2} S}{C_{H}}\right) .
\end{aligned}
$$

The resulting solution is a supersymmetric AdS vacuum in which both the four-dimensional dilaton $\varphi$ and the fourdimensional volume modulus $\rho$ have been stabilized. We will defer our discussion of the physics in $E_{8}^{(1)}$ to Sec. VC.

\section{B. Stabilization of multiple Kähler moduli}

On a threefold $X$ with $h^{1,1}>1$ Kähler moduli, the formulas of the preceding section can be generalized:

$$
f_{(i)}^{W}=S+\beta_{i}^{\alpha} T_{\alpha},
$$

where $i=1,2$ labels the gauge groups $E_{8}^{(1)}, E_{8}^{(2)}$ and $\alpha$ $=1, \ldots, h^{1,1}$ indexes the independent Kähler moduli.

We will need to define a few quantities related to the generators $J^{\alpha}$ of $H^{1,1}(X, Z)$ :

$$
\begin{aligned}
\beta_{1}^{\alpha} & \equiv \frac{1}{8 \pi^{2}} \int_{X} J^{\alpha} \wedge\left[c_{2}\left(V_{1}\right)-c_{2}\left(V_{2}\right)\right], \\
\beta_{2}^{\alpha} & \equiv \frac{1}{8 \pi^{2}} \int_{X} J^{\alpha} \wedge\left[c_{2}\left(V_{2}\right)-c_{2}\left(V_{1}\right)\right], \\
c_{\alpha \beta \gamma} & \equiv \int_{X} J^{\alpha} \wedge J^{\beta} \wedge J^{\gamma} .
\end{aligned}
$$

\footnotetext{
${ }^{13}$ Notice that we are putting more instantons in the hidden sector than in the observable sector, which is a somewhat unusual situation compared to the bulk of the literature.
}

The $c_{\alpha \beta \gamma}$ are the intersection numbers of $X$.

The Kähler potential (2.23) now takes the form

$$
\begin{aligned}
\mathcal{K}= & -\log (S+\bar{S})-\log \left(c_{\alpha \beta \gamma} \mathcal{T}^{\alpha} \mathcal{T}^{\beta} \mathcal{T}^{\gamma}\right) \\
& -\log \left(-\frac{i}{4 \alpha^{\prime 3}} \int \Omega \wedge \bar{\Omega}\right),
\end{aligned}
$$

with $2 \mathcal{T}^{\alpha} \equiv T^{\alpha}+\bar{T}^{\alpha}$, while the complete superpotential, including hidden-sector gaugino condensation, is

$$
\frac{W}{\mu^{3}}=\frac{2 h}{c}-C_{H} \exp \left(-\frac{8 \pi^{2}}{C_{H}}\left(S+\beta^{\alpha} T_{\alpha}\right)\right) .
$$

This superpotential depends nontrivially on the dilaton and on all the Kähler moduli.

In order to find a supersymmetric solution we will assume that all the $\beta^{\alpha}$ are nonzero. Combining (5.15) and (5.14) and imposing $W_{; S}=W_{; T}=0$, we find

$$
\begin{aligned}
& S \frac{\partial}{\partial T_{\delta}}\left(c_{\alpha \beta \gamma} \mathcal{T}^{\alpha} \mathcal{T}^{\beta} T^{\gamma}\right) \\
& \quad=\beta_{2}^{\delta}\left(c_{\alpha \beta \gamma} \mathcal{T}^{\alpha} \mathcal{T}^{\beta} \mathcal{T}^{\gamma}\right), \\
& h=\left(\frac{C_{H} c}{2}+8 c \pi^{2} \operatorname{Re}(S)\right) \exp \left(-\frac{32 \pi^{2} S}{C_{H}}\right),
\end{aligned}
$$

where the second relation is identical to (5.9).

The result is a supersymmetric AdS vacuum without moduli. To recapitulate, we have now seen that the combination of fractional flux with a gaugino condensate can stabilize the complex structure moduli, the Kähler moduli, and the dilaton.

\section{A strong-coupling problem}

We have just seen that the potential for the dilaton and Kähler moduli has a supersymmetric AdS minimum whose location is given, in the case of one Kähler modulus, by (5.8) and (5.9). However, there is an evident problem with this minimum. Suppose that some subgroup of $E_{8}^{(1)}$ remains unbroken at low energies. The naive $E_{8}^{(1)}$ gauge coupling function, $f_{1}=S-\beta T$, appears to be negative, $f_{1}=-2 S$. Moreover, one might think that before becoming negative, $f_{1}$ must pass through zero, at which point one encounters a singularity where the gauge coupling diverges.

It is clear a priori that such a problem cannot exist in the full theory. Moduli (and parameter) spaces of fourdimensional supersymmetric theories are complex and hence can only have singularities at complex codimension one. It follows that one can always continue around any point of naively singular gauge coupling, obtaining a unitary theory with positive $g^{2}$ on the "other side." Numerous examples of 
such phenomena have been explored in various fourdimensional supersymmetric gauge theories over the past several years, most recently in interpreting the $G_{2}$ flop in Ref. [45].

In fact, what we are encountering here is (at least in those cases that are most easily understood) a close relative of the well-studied strong coupling transitions in six-dimensional string vacua with $(0,1)$ supersymmetry [46]. The observable sector gauge coupling diverges precisely when the ratio $S / T$ reaches a fixed value; this is in fact a point in moduli space where an effective six-dimensional coupling is becoming strong. As explained in Ref. [46], in dual type II or F-theory descriptions, this phenomenon can be modeled locally in terms of a geometric transition that affects the D-branes or local geometry responsible for $E_{8}^{(1)}$. On the other side of the geometric transition, the $E_{8}^{(1)}$ physics remains sensible, and there is a new effective description of the low-energy gauge theory.

In the remainder of this subsection we investigate this strong-coupling singularity. The resolution is necessarily model dependent, so we simply review some dual descriptions that shed light on the phenomenon, and give an explicit example where the physics on the "other side" of the transition is fully understood. Of course in as much as one wishes to embed the standard model in $E_{8}^{(1)}$, it would be crucial to have a good dual description of this new phase. For readers who find this too daunting a challenge, we can only suggest that the special case $\beta_{1,2}=0$ neatly sidesteps the issue, leaving a no-scale model with an unfixed volume modulus. However, we emphasize that more generally, the only assumption we really need to make is that the physics of the transition does not introduce new terms in the superpotential. For models where $E_{8}^{(1)}$ is broken to a low-energy field theory that does not dynamically generate a superpotential, this is quite plausible.

\section{Dual descriptions of the strong-coupling singularity}

The appearance of strong gauge coupling in heterotic models with nonzero $\beta$ is well known. The problem is easily seen in compactifications of heterotic M-theory to four dimensions, where it manifests as a linear shrinking [47] of the Calabi-Yau volume as a function of location on the M-theory interval. For some critical size of the interval, the Calabi-Yau manifold has zero volume at one boundary, rendering the supergravity approximation invalid.

A closely related problem arises in compactifications of the $E_{8} \times E_{8}$ heterotic string on $\mathrm{K} 3 \times T^{2}$. The gauge bundle in such a model is specified in part by a choice of instanton numbers $(12-n, 12+n)$ in the two $E_{8}$ 's. If $n$ is positive then the first $E_{8}$ is more strongly coupled than the second; this is analogous to positive $\beta$ in our models. At a finite value of the heterotic dilaton the first $E_{8}$ has infinite gauge coupling.

This configuration is dual to compactification of type IIA string theory on a Calabi-Yau threefold that is an elliptic fibration over the Hirzebruch surface $F_{n}$. Recall that $F_{n}$ has a single curve of self-intersection $-n$. The volume of this curve is dual to the heterotic dilaton in such a way that shrinking the $-n$ curve to zero volume coincides with infi- nite gauge coupling in the first $E_{8}$. This suggests that one could use the type II geometry to understand the nature of the strong-coupling singularity. While this approach is rather complicated for general $n$ (see, e.g., Ref. [46] for work in this direction), we will see that the case $n=1$ is relatively straightforward.

It is important to remember that type II strings on such a Calabi-Yau threefold yield $\mathcal{N}=2$ supersymmetry in four dimensions, twice as much as the models we have considered in this paper. This greatly facilitates analysis of the singularity, in particular because the geometry can be described via a prepotential. A direct study of the $\mathcal{N}=1$ system would be more challenging, but we expect the generic features, including the positive gauge coupling function, to be similar in the two cases. One would simply have to study the geometry of a dual F-theory compactification on a Calabi-Yau fourfold, instead of type II strings on a Calabi-Yau threefold.

\section{A simple flop model of the strong-coupling singularity}

We will now construct a simple model in which, in a sense that we will make precise, the gauge kinetic term $f_{1}$ undergoes a flop. Recall that in the flop of a curve, the volume of the curve vanishes on a wall of the Kähler cone. ${ }^{14}$ However, instead of continuing to negative values on the far side, the volume is actually positive in the new Kähler cone. In certain $\mathcal{N}=2$ heterotic-type IIA dual pairs [50], the singularity in the Calabi-Yau prepotential when a curve in the type IIA geometry undergoes a flop (and an effective gauge coupling becomes singular) is dual to a heterotic strongcoupling singularity. We describe one such example below. It is important to stress that as expected on completely general grounds, the effective $g^{2}$ remains positive everywhere in the properly interpreted type II moduli space.

The examples we have in mind, and their heterotic duals, are well known. Our presentation of a specific example will closely follow [51], which mapped out in detail several heterotic-type II dual pairs.

Let $X$ be the Calabi-Yau threefold space, which is an elliptic fibration over $F_{1}$. The prepotential for the Kähler moduli space of $X$ is [51]

$$
\mathcal{F}_{\mathrm{II}}=\frac{4}{3} t_{1}^{3}+\frac{3}{2} t_{1}^{2} t_{2}+\frac{1}{2} t_{1} t_{2}^{2}+t_{1}^{2} t_{3}+t_{1} t_{2} t_{3}
$$

where $t_{i}$ are the Kähler moduli. The volume of the -1 curve is controlled by $t_{3}$. One can find a set of dual heterotic variables $S, T, U$, which are related to the type II variables by

$$
t_{1}=U, \quad t_{2}=T-U, \quad t_{3}=S-\frac{T}{2}-\frac{U}{2} .
$$

In heterotic variables, the prepotential reads

$$
\mathcal{F}_{h}=S T U+\frac{1}{3} U^{3}
$$

\footnotetext{
${ }^{14}$ In the full physical theory the volume is complexified, and one can go "around" the wall of the Kähler cone by turning on a nonzero $\theta$ angle $[48,49]$.
} 
We know that the type II operation of shrinking the -1 curve corresponds to strong gauge coupling in the heterotic picture. This instructs us to identify $S-T / 2-U / 2$ with the visiblesector gauge coupling. ${ }^{15}$

Now, to study the effect of the strong gauge coupling, we flop the curve corresponding to $t_{3}$. The fields transform as

$$
\left(t_{1}, t_{2}, t_{3}\right) \rightarrow\left(t_{1}+t_{3}, t_{2}+t_{3},-t_{3}\right),
$$

leading to the prepotential for $\tilde{X}$, the image of $X$ under the flop. It turns out that $\widetilde{X}$ is not a K3 fibration, and furthermore it is not dual to a perturbative heterotic model.

Given this linear implementation of the flop in type II variables, we can apply this transformation to the heterotic variables (3.9). This yields

$$
\begin{aligned}
& \left(U, T-U, S-\frac{T}{2}-\frac{U}{2}\right) \\
& \quad \rightarrow\left(S+\frac{U}{2}-\frac{T}{2}, S+\frac{T}{2}-\frac{3 U}{2}, \quad \frac{T}{2}+\frac{U}{2}-S\right) .
\end{aligned}
$$

The key result is that the visible-sector gauge coupling has changed sign,

$$
S-\frac{T}{2}-\frac{U}{2} \rightarrow-S+\frac{T}{2}+\frac{U}{2}
$$

In this new Kähler cone, the visible-sector coupling is sensible provided $T+U>2 S$, which is complementary to the initial restriction $T+U<2 S$.

We have therefore seen that in this very simple example, the gauge coupling function for the visible sector is sensible and positive on both sides of the strong-coupling transition. We expect this result to hold in all of the cases of interest, simply from macroscopic arguments about supersymmetric theories. It would be interesting to generalize the simple illustration above to $\mathcal{N}=1$ heterotic vacua by studying the dual geometric transitions in F-theory compactifications on Calabi-Yau fourfolds.

\section{Fractional invariants and weak coupling}

Let us now determine the conditions under which the stable vacuum exists at modestly large values of $S$ and $T$. Note that this does not mean that all of the physics is weakly coupled, since as we just discussed, we have undergone a strong-coupling transition in $E_{8}^{(1)}$. However, some other sectors of the theory may remain perturbative at large $S$ and $T$, so it is still of interest to know that stabilization at large $S$ and $T$ is possible.

The goal is to arrange that the volume of the Calabi-Yau manifold is large in string units, while the string coupling is

\footnotetext{
${ }^{15}$ To make contact with our earlier notation, $T$ and $U$ are the two $T^{\alpha}$, and $\beta_{2}=\frac{1}{2}$ for $\alpha=1,2$.
}

small: ${ }^{16}$

$$
\begin{aligned}
& (S T)^{1 / 8}=e^{\sigma}>1, \\
& \left(\frac{T^{3}}{S}\right)^{1 / 2}=e^{\phi}<1 .
\end{aligned}
$$

Recall that $\phi$ is the ten-dimensional dilaton; we denote the four-dimensional dilaton by $\varphi$. Using the relation (5.8), we have

$$
\begin{aligned}
& \left(\frac{3}{\beta}\right) S^{2}=e^{8 \sigma}, \\
& \left(\frac{3}{\beta}\right)^{3} S^{2}=e^{\phi} .
\end{aligned}
$$

Clearly $\beta>3$ is a necessary condition for perturbative validity. It follows from (5.3) that this condition can only be met if the bundle $V_{2}$ is nontrivial; hence gaugino condensation in an unbroken hidden-sector $E_{8}$ is not compatible with this method of volume stabilization. To see explicitly that large $\beta$ is possible within known constructions we refer to the plots of Ref. [52].

From the form of the solution (5.9) it is clear that the values of $S$ and $T$ at the stable minimum increase as the Chern-Simons invariant becomes smaller. We are therefore interested in finding three-cycles admitting extremely small Chern-Simons invariant.

Small values of the Chern-Simons invariant are distasteful but not unattainable. We saw in Sec. III that it is possible to get a small Chern-Simons invariant $h$ by working on a Calabi-Yau manifold that has a three-cycle $Q$ satisfying

$$
\pi_{1}(Q)=Z_{p}
$$

for $p \gg 1$. The simplest example of this is a Lens space. One way to generate even smaller $h$ is to take $Q$ to be a general Seifert manifold $\Sigma\left(a_{1}, \ldots, a_{n}\right)$, since the minimal value of $h$ would scale like

$$
h^{-1} \sim \prod_{i=1}^{n} a_{i} .
$$

With several $a_{i}$ one could then generate very small fractional fluxes.

\section{E. Summary of requirements}

Let us briefly review the conditions on the Calabi-Yau manifold $X$ and the gauge bundles $V_{i}$ that ensure the existence of the supersymmetric vacuum (5.9) with both dilaton and Kähler moduli stabilized. Conditions essential to the mechanism are listed first, while those related to detailed model building come last.

\footnotetext{
${ }^{16}$ For simplicity we now present the formulas for the case of one Kähler modulus, the overall volume; the generalization is straightforward.
} 
(1) In order to achieve a small value of the three-form flux, the Calabi-Yau manifold $X$ must have a nontrivial fundamental group and must admit gauge connections that have a fractional Chern-Simons invariant on a three-cycle $Q$ that is not torsion. One of the bundles $V_{1}, V_{2}$ must then be chosen to have such a gauge connection, i.e., suitable Wilson lines. These conditions are automatically met in a large class of realistic string models.

(2) For gaugino condensation to be possible in $H \subset E_{8}^{(2)}$, the bundle $V_{2}$ must break $E_{8} \rightarrow H$ without introducing any light charged matter, leaving a pure gauge group. For example, if $H=E_{7}$ then there is no index theorem protecting charged matter transforming in the $\mathbf{5 6}$, so we expect that this condition is generically satisfied. If instead $H=E_{6}$ the number of chiral generations is $\left|\frac{1}{2} \int_{X} c_{3}\left(V^{(2)}\right)\right|$. The bundle $V_{2}$ should be chosen so that this vanishes. ${ }^{17}$

(3) In order to stabilize the overall volume we must choose bundles for which the quantity $\beta_{2}$ defined in (5.3) is nonzero. To stabilize multiple Kähler moduli we must take all of the $\beta_{2}^{\alpha}$ to be nonzero. To ensure stabilization of the volume above the string scale, we should also have $\beta_{2}>3$, with an analogous condition for the case of many moduli.

(4) If the Kähler moduli are to be stabilized, the initial configuration and the final stable minimum are on opposite sides of a transition in which the visible sector becomes strongly coupled. It follows that the visible-sector gauge theory can only be properly understood in models where this strong-coupling transition can be followed in detail. Better understanding of this transition is a necessary prelude to the building of realistic models. Readers uncomfortable with the transition are advised to set $\beta_{1}=\beta_{2}=0$, in which case one is left with a no-scale model with fixed dilaton and an unfixed volume modulus.

(5) Further constraints will be necessary to obtain realistic low-energy physics. For example, $V_{1}$ should contain appropriate Wilson lines that break the visible-sector GUT to the Standard Model gauge group. (It is sometimes possible to arrange that these same Wilson lines also provide the fractional Chern-Simons invariant.) The vacua we have constructed have negative cosmological constant, with an energy density not far below the string scale. This must certainly be modified to lead to a sensible cosmological model. Finally, if we wish to stabilize at very weak coupling then the fundamental group of the Calabi-Yau manifold must be unusually large.

Clearly, the greatest obstacle to calculability in this scenario is the strong-coupling transition in the observable sector. It is conceivable that one could avoid this difficulty by combining fractional Chern-Simons invariants and gaugino condensation with a non-Kähler compactification geometry, for in this case the volume modulus can be stabilized at tree level. However, for the bulk of our analysis, the only real

\footnotetext{
${ }^{17}$ One could imagine other possibilities in which charged matter in the hidden sector generates a nonperturbative superpotential that can be used for stabilization. See, e.g., Ref. [53] for a discussion of this possibility in the context of racetrack models.
}

assumption we have made is that the unknown physics of the visible sector does not modify the superpotential. This seems believable provided that the low-energy $\mathcal{N}=1$ gauge theory that emerges from $E_{8}^{(1)}$ is not one that dynamically generates a superpotential.

\section{DUALITY TO TYPE IIA AND M THEORY}

The models studied in this paper are related by various dualities to a particular class of $\mathcal{N}=1$ compactifications of $\mathrm{M}$ theory and type IIA string theory. These models have recently received some attention due in part to phenomenological applications (see, e.g., Refs. [13,54-60]). After appropriate duality transformations our mechanism for moduli stabilization can be applied to these models as well. In this section we briefly discuss various aspects of these dualities, as well as their implications.

\section{A. Heterotic-type IIA duality}

Our considerations have thus far been limited to the $E_{8}$ $\times E_{8}$ heterotic string, but the discussion can be repeated almost verbatim for the $\operatorname{Spin}(32) / Z_{2}$ heterotic string compactified on a Calabi-Yau manifold. The latter theory is related to an $\mathcal{N}=1$ compactification of type IIA string theory by the following chain of dualities:

$$
\begin{gathered}
\operatorname{Spin}(32) / \mathbb{Z}_{2} \quad \stackrel{S}{\text { Het. }} \stackrel{\leftrightarrow}{\leftrightarrow} \text { type } \mathrm{I} \leftrightarrow \text { type } \operatorname{IIB} / \Omega \\
\stackrel{T}{\leftrightarrow} \text { type } \operatorname{IIA} /(\Omega \cdot \mathcal{I}) .
\end{gathered}
$$

Let us now explain each step in this duality in more detail and, in particular, find the relation between the parameters and the coupling constants. The first relation is the standard strong-coupling-weak-coupling duality between the $\operatorname{Spin}(32) / Z_{2}$ heterotic string theory and type I string theory. The effective supergravity action in the latter theory is similar to the heterotic supergravity action, with the type I and heterotic variables related by

$$
\begin{gathered}
\phi_{\mathrm{I}}=-\phi_{H}, \\
g_{M N}^{I}=g_{M N}^{H} e^{-\phi_{H} .}
\end{gathered}
$$

At the next step in the chain of dualities (6.1) we identify type I string theory with an orientifold of type IIB closed string theory, where $\Omega$ denotes the worldsheet parity symmetry. The parameters and the coupling constants in the supergravity action do not change under this identification, although some terms acquire a different interpretation. In particular, in the type IIB theory the gauge degrees of freedom arise as open string states on the world-volume of 32 space-filling D9-branes. Thus, the Wilson lines of the original heterotic string theory become Wilson lines on D9branes, and the ten-dimensional gauge coupling is simply

$$
\frac{g_{10}^{2}}{\alpha^{\prime 3}}=e^{\phi_{\mathrm{I}}}=e^{\phi_{\mathrm{IIB}}}
$$


From (6.2) and (6.4) we find

$$
\phi_{\mathrm{IIB}}=\phi_{\mathrm{I}}=-\phi_{H}
$$

The last step in (6.1) is the $T$ duality-mirror symmetry, to be more precise-between type IIB string theory on a Calabi-Yau manifold $X$ and type IIA theory on the mirror manifold $\widetilde{X}$. Strictly speaking, the dual background is an orientifold of $\widetilde{X}$, where the involution changes the orientation of the $T^{3}$ fibers. Under $T$ duality, the space-filling D9branes transform into D6-branes wrapped over the base, $Q$, of the special Lagrangian torus fibration [29]. The parameters of the resulting type IIA background can be obtained from the usual $T$-duality rules:

$$
\phi_{\mathrm{IIA}}=\phi_{\mathrm{IIB}}-\log \left(\frac{V_{X}^{\mathrm{IIB}}}{V_{Q}^{\mathrm{IIB}} \alpha^{\prime 3 / 2}}\right)=\frac{1}{2} \phi_{H}-\log \left(\frac{V_{X}^{H}}{V_{Q}^{H} \alpha^{\prime 3 / 2}}\right) \text {. }
$$

Here $V_{X}$ and $V_{Q}$ denote, respectively, the volume of the Calabi-Yau space $X$ and the volume of the base threemanifold $Q$ in the string theory given by the superscript.

To summarize, after a chain of dualities (6.1) we found that our heterotic string models are dual to IIA string theory on a mirror Calabi-Yau manifold $\widetilde{X}$, with D6-branes wrapped over the special Lagrangian three-cycle $Q$. This is precisely the configuration studied in Refs. $[55,13,56,57]$. In these papers, $Q$ is usually taken to be a Lens space, $Q=\mathbf{S}^{3} / \mathbb{Z}_{p}$, and the Calabi-Yau manifold $\widetilde{X}$ is usually assumed to be noncompact. If $\widetilde{X}$ is compact, as described above, then the presence of orientifold six-planes is crucial to cancel the D6-brane charge.

Observe that on the D6-brane world-volume there is a topological coupling between the gauge field, $F=d A$ $+A \wedge A$, and the Ramond-Ramond tensor fields, $C=C_{1}$ $+C_{3}+\cdots$,

$$
\operatorname{tr} \int_{\mathbf{R}^{4} \times Q} C \wedge e^{F}
$$

Among other terms, this expression contains a coupling

$$
C S(A, Q) \int_{\mathbf{R}^{4}} G,
$$

which we obtained by expanding (6.7) and integrating by parts. It follows that D6-branes wrapped over $Q$ with a nonzero value of the Chern-Simons invariant act as an effective source for the Ramond-Ramond four-form field strength in the four uncompactified directions.

\section{Comments on proton decay}

Using the chain of dualities (6.1) we have now related our setup to compactifications of type IIA string theory, where the GUT gauge theory is realized on the world-volume of D6-branes wrapped over a compact three-manifold $Q$. Similar configurations have been discussed in a recent work of
Klebanov and Witten [57] (see also Ref. [61]), where it was shown that the proton decay rate from dimension six operators is given by ${ }^{18}$

$$
A_{\mathrm{IIA}} \sim \frac{g_{\mathrm{YM}}^{4 / 3} L(Q)^{2 / 3} e^{\phi_{\mathrm{IIA}} / 3}}{M_{\mathrm{GUT}}^{2}},
$$

where $g_{\mathrm{YM}}$ is the GUT gauge coupling, and $M_{\mathrm{GUT}}$ is the unification scale. This scale is determined by the size of the three-manifold $Q$,

$$
M_{\mathrm{GUT}}=\left(\frac{L(Q)}{V_{Q}}\right)^{1 / 3},
$$

where the extra factor $L(Q)$ accounts for the one-loop threshold corrections from Kaluza-Klein harmonics on $Q$ [56,57]. Specifically, $L(Q)$ is a topological invariant of $Q$, known as the Ray-Singer torsion.

Let us now compute the proton decay rate in our heterotic models. In contrast to the result of Ref. [57], we expect in our case the conventional amplitude

$$
A_{h} \sim \frac{g_{\mathrm{YM}}^{2}}{M_{\mathrm{GUT}}^{2}} \sim \alpha^{\prime} e^{\phi / 2+2 \sigma},
$$

where the unification scale and the gauge coupling are given by (2.9) and (2.21), respectively. By tracing the chain of dualities (6.1) in reverse, being careful to include the constant rescaling of the Einstein-frame metric mentioned in Sec. II, one can verify that (6.9) and (6.11) differ by the factor $\alpha_{\mathrm{GUT}}^{1 / 3} e^{-\phi_{\mathrm{IIA}} / 3}$, which exhibits the anomalous scaling with $\alpha_{\mathrm{GUT}}$ explained in Ref. [57].

\section{B. Lift to M-theory}

Now let us consider the M-theory lift of the type IIA configuration considered above. Since D6-branes wrapped over a special Lagrangian submanifold $Q \subset \widetilde{X}$ preserve $\mathcal{N}$ $=1$ supersymmetry in four dimensions, their lift to $\mathrm{M}$ theory must be described by a seven-dimensional manifold $X_{G_{2}}$ with $G_{2}$ holonomy. Topologically, $X_{G_{2}}$ can be viewed as a K3 fibration over $Q$ [62],

$$
\begin{gathered}
\mathrm{K} 3 \rightarrow X_{G_{2}}, \\
\downarrow \\
Q
\end{gathered}
$$

such that each K3 fiber has an ADE singularity, which corresponds to the type of the gauge group on the D6-branes. For example, $\mathrm{SU}(5)$ gauge theory would lift to a $G_{2}$ manifold with $A_{4}$ singularities in the fiber. The dual M-theory geometry (6.12) can be obtained directly from the heterotic string theory on a Calabi-Yau manifold $X$ by using the familiar duality between $\mathrm{M}$ theory on $\mathrm{K} 3$ and heterotic string theory on $T^{3}$. Applying this duality to each fiber in the special Lagrangian torus fibration, $X \rightarrow Q$, we end up with $\mathrm{M}$ theory on a seven-manifold $X_{G_{2}}$ with $G_{2}$ holonomy and to-

\footnotetext{
${ }^{18}$ For simplicity, we omit numerical factors of order one.
} 
pology (6.12). Various aspects of M theory on $G_{2}$ manifolds of this kind have been studied in Refs. [54,55,63,64,13,56].

Now let us consider a D6-brane configuration with nontrivial gauge fields characterized by $C S(A, Q) \neq 0$. According to (6.8), such gauge fields act as a source (localized on the three-cycle $Q$ ) for the space-time component of the fourform flux, $G_{0123}$. In the effective four-dimensional field theory, this means there is a nonzero superpotential induced by $C S(A, Q)$. In $\mathrm{M}$ theory, the relevant interaction term (6.8) appears due to anomaly inflow at the location of ADE singularities [65], while the effective superpotential is generated by topologically nontrivial gauge fields supported at the singularities [58].

The models studied in this paper have real values of the Chern-Simons invariant $C S(A, Q)$. However, Acharya has argued [58] that, in a more general setting, the superpotential induced by gauge fields should be given by a complex Chern-Simons invariant. A deeper understanding of the connection between these ideas would be quite interesting.

\section{DOMAIN WALLS}

In order to obtain an expression for the effective superpotential of an $\mathcal{N}=1$ supersymmetric gauge theory, it is often useful to study the spectrum of BPS domain walls. Moreover, in a theory with gaugino condensation, the domain walls provide information about the breaking of chiral symmetry and about other phenomena of interest.

With this motivation in mind, let us consider domain walls in our models, ${ }^{19}$ where different vacua are characterized by the values of the Chern-Simons functional, $C S(A, Q)$. Hence, the BPS domain walls are represented by self-dual field configurations (instantons) supported on $Q$ $\times \mathbf{R}$, where $\mathbf{R}$ represents a spatial direction orthogonal to the domain wall. Since $C S(A, Q)$ takes fractional values, such instantons carry fractional charge,

$$
c_{2}=-\frac{1}{8 \pi^{2}} \int \operatorname{tr}(F \wedge F)=\left.C S(A, Q)\right|_{-\infty}-\left.C S(A, Q)\right|_{+\infty} .
$$

The instanton action is given by $\int_{Q \times \mathbf{R}} \operatorname{tr}\left(F \wedge^{*} F\right)$, which, using the self-duality of the gauge field $F$, can be written as

$$
\int_{Q \times \mathbf{R}} \operatorname{tr}(F \wedge F) .
$$

Furthermore, using (7.1) one can rewrite the instanton action as the difference of the values of the Chern-Simons functional, $\triangle C S(A, Q)$. Comparing this formula with the standard expression for the tension of a domain wall in $\mathcal{N}=1$ supersymmetric theory, $T=|\Delta W|$, we come to our previous result (2.25) for the effective superpotential induced by nontrivial gauge fields $[23,24]$ :

\footnotetext{
${ }^{19}$ For a related discussion see also Refs. [66,67].
}

$$
W_{\text {flux }}=\int_{Q} \Omega_{3}(A) .
$$

Now let us consider the degeneracy of domain walls interpolating between two vacua with fractional Chern-Simons functional, $C S(A, Q)$, for some three-cycle $Q \subset X$. At least in the classical theory, the BPS domain walls come in continuous families. Specifically, the moduli space of domain walls with fractional charge $c_{2}$ is isomorphic to the moduli space of charge- $c_{2}$ instantons on $Q \times \mathbf{R}$,

$$
\mathcal{M}\left(Q \times \mathbf{R} ; c_{2}\right) .
$$

Without loss of generality, we can study SU(2) instantons and, for concreteness, take $Q$ to be a Lens space,

$$
Q=\mathbf{S}^{2} / \mathbb{Z}_{p}
$$

Then, according to (3.14), the Chern-Simons functional on $Q$ can take the fractional values

$$
C S(A, Q)=-\frac{m^{2}}{4 p}-\frac{\lambda^{2}}{2} .
$$

Here we follow the notations of Ref. [68], introduced at the end of Sec. III, where $m$ is an integer defined modulo $2 p$.

Consider an instanton on $Q \times \mathbf{R}$ that interpolates between different values of the Chern-Simons invariant $C S(A, Q)$. According to (7.1) and (7.4), such an instanton connects two states characterized by different rotation numbers $m$ and $m^{\prime}$ $=m \bmod 2$, and carries a fractional instanton charge $c_{2}$ $=k / p$. Put differently, it is described by a triplet of integers, $\left(k, m, m^{\prime}\right)$. Following Ref. [68], let us express $\left(m, m^{\prime}\right) \sim(a$ $-b, a+b)$ in terms of $a$ and $b$, such that

$$
\begin{aligned}
& a=\left(m^{\prime}+m\right) / 2 \bmod p, \\
& b=\left(m^{\prime}-m\right) / 2 \bmod p .
\end{aligned}
$$

Using the above expression (7.4) for the value of the Chern-Simons functional, we find the corresponding instanton number:

$$
\begin{aligned}
c_{2} & =\left.C S(A, Q)\right|_{-\infty}-\left.C S(A, Q)\right|_{+\infty} \\
& =-\frac{(a-b)^{2}}{4 p}+\frac{(a+b)^{2}}{4 p}=\frac{a b}{p} .
\end{aligned}
$$

Therefore, we have

$$
k=a b \bmod p .
$$

Now we are in a position to describe the moduli space, $\mathcal{M}$, of instantons on $Q \times \mathbf{R}$ that interpolate between gauge connections with rotation numbers $m=a-b$ and $m^{\prime}=a$ $+b$. Since instanton configurations always have a modulus that represents their position in $\mathbf{R}$, it makes sense to divide by translations and consider the reduced moduli space,

$$
\mathcal{M}^{\prime}=\mathcal{M} / \mathbf{R} \text {. }
$$


TABLE I. $\operatorname{Dim}\left(\mathcal{M}^{\prime}\right)$ for the Lens space, $Q=\mathbf{S}^{3} / Z_{5}$.

\begin{tabular}{lccccc}
\hline \hline$b$ & & & & & \\
\hline & 0 & 1 & 2 & 3 & 4 \\
\hline 0 & - & - & - & - & - \\
1 & - & 0 & 2 & 2 & 2 \\
2 & - & 2 & 4 & 6 & 10 \\
3 & - & 2 & 6 & 12 & 18 \\
4 & - & 2 & 10 & 18 & 24 \\
\hline \hline
\end{tabular}

Using index theorems one can compute the virtual dimension of the reduced moduli space [68],

$$
\begin{aligned}
\operatorname{Dim}\left(\mathcal{M}^{\prime}\right)= & \frac{8 k}{p}-4+n \\
& +\frac{2}{p} \sum_{j=1}^{p-1} \cot ^{2} \frac{\pi j}{p}\left(\sin ^{2} \frac{\pi j m}{p}-\sin ^{2} \frac{\pi j m^{\prime}}{p}\right),
\end{aligned}
$$

where $n \in\{0,1,2\}$ is the number of $m, m^{\prime} \neq 0, p$. It turns out that this virtual dimension is always even. In order to illustrate this general formula, in Table I we list the dimensions of the moduli spaces of fractional charge instantons on $\mathbf{S}^{3} / Z_{5} \times \mathbf{R}$. In terms of $a$ and $b, m=a-b, m^{\prime}=a+b$, and the instanton number $k=a b \bmod 5$.

The dimension of the moduli space tends to grow with the instanton number, $k=a b$. For low values of the dimension, one can describe $\mathcal{M}^{\prime}$ rather explicitly using general topological properties [68] (see also Refs. [69,70]). When $\operatorname{Dim}\left(\mathcal{M}^{\prime}\right)=0$, the reduced moduli space must be just a point. In this case, we have only one domain wall interpolating between two vacua. Furthermore, the Euler number of $\mathcal{M}^{\prime}$ is given by the number of solutions $(a, b)$ to Eqs. (7.5) such that $a b=k$. In particular, this implies that

$$
\chi\left(\mathcal{M}^{\prime}\right) \geqslant 0 .
$$

Hence, when $\operatorname{Dim}\left(\mathcal{M}^{\prime}\right)=2$, the reduced moduli space must be of the form

$$
\mathcal{M}^{\prime}=\mathbf{S}^{2} \backslash F
$$

where $F$ is a set of 0,1 , or 2 points.

For example, let us take $p=5, a=2$, and $b=1$. This implies $k=2, m=1$, and $m^{\prime}=3$. Then, from Table I we find that $\mathcal{M}^{\prime}$ must be of real dimension 2, and by looking at the Euler number $\chi\left(\mathcal{M}^{\prime}\right)=2$ one concludes that in this example the moduli space is simply a two-sphere,

$$
\mathcal{M}^{\prime}=\mathbf{S}^{2} \text {. }
$$

Since this space is compact, we expect that the degeneracy of domain walls of charge $c_{2}=\frac{2}{5}$ interpolating between vacua with $m=1$ and $m^{\prime}=3$ is given by the cohomology of $\mathcal{M}^{\prime}$. Therefore, in this example we find

$($ Number of domain walls $)=2$.
The above results suggest the following conjecture for the degeneracy of domain walls with small fractional charge, $c_{2}=k / p$,

$$
\text { (Number of domain walls) }=\left\{\begin{array}{ll}
1 & \text { if } k=1 \\
2 & \text { if } k=2
\end{array}\right. \text {. }
$$

In other words, we expect that there is always only one domain wall of the minimal fractional charge, whereas the degeneracy of domain walls with twice the minimal charge is equal to 2 . It would be interesting to pursue this analysis further

\section{DISCUSSION}

We have argued that it is possible to stabilize the complex structure moduli, Kähler moduli, and dilaton of heterotic Calabi-Yau compactifications. Our ingredients are hiddensector gaugino condensation combined with a flux-generated superpotential arising from a flat connection with fractional Chern-Simons invariant. For the non-Kähler compactifications of Refs. [41,42] our result looks even more promising, since there the volume is stabilized at tree level, and the only concern is the dilaton.

One omission from our list of stabilized moduli is the vector bundle moduli. Following the analysis in Ref. [71], it seems likely that the very existence of bundle moduli is not generic. Massless modes arising from the moduli of a vector bundle $V$ are associated with elements of the group $H^{1}(X$, End $(V))$. Typically there is no index theorem that allows one to argue that this group should be nontrivial. Even if the group were nontrivial, a generic infinitesimal deformation of the vector bundle is obstructed at some finite order and so does not constitute a modulus. ${ }^{20}$

In addition to the omission of a detailed discussion of bundle moduli, we have used standard approximations in describing the hidden-sector gaugino condensation. For instance, in real string models, the hidden sector would have massive fields charged under the hidden $E_{8}$. This would lead to corrections to the form of the superpotential used here, which presumably arise as more highly damped exponentials in $S$. While for reasonable values of $\operatorname{Re}(S)$ this should not be a large correction, it would be nice to have exact results. These are not yet available for $\mathcal{N}=1$ supersymmetric compactifications of heterotic strings.

The solutions we have constructed are supersymmetric AdS vacua. It is natural to ask whether one can add a source of supersymmetry-breaking energy which lifts these models to de Sitter vacua, along the lines of Ref. [40]. In fact, there are significant similarities between the type IIB constructions

\footnotetext{
${ }^{20}$ Nevertheless, simple bundles constructed by mere humans often have moduli. In many such simple cases, even nonperturbative sigma model effects do not suffice to lift them [72]. Examples of superpotentials arising for the bundle moduli associated with small instantons in heterotic M-theory are described in, e.g., Ref. [73].
} 
of Ref. [40] and the heterotic models discussed here. As noted in Sec. IV, the superpotential in each case consists of a small, constant term from flux and an exponential term from nonperturbative gauge dynamics. To continue this analogy and include supersymmetry breaking, one would have to introduce the heterotic dual of the anti-D3-brane introduced in Ref. [40]. In heterotic M-theory this would correspond to a nonsupersymmetric wrapped M5-brane. To achieve control over the construction, one would need to introduce such an object in a heterotic background with significant warping.

Burgess, Kallosh, and Quevedo [74] have recently proposed that a Fayet-Iliopoulos $D$-term potential could serve as another useful source of energy for uplifting heterotic models. The stable AdS vacua we have discussed would appear to be a suitable setting for such a mechanism, but we leave the construction of explicit models as a subject for future exploration. Again, one would have to arrange for a suitably small $D$-term to justify the analysis.

The present proposal for manufacturing vacua without moduli, combined with the constructions in Refs. [40,58, 74-76], is a small step towards filling out our picture of the "discretuum" [77] of string or M-theory vacua. This is the full space of vacua of string theory, including all of the possibilities for the background fluxes, wrapped branes, and other discrete data. Interesting general aspects of this landscape of string theory vacua have recently been discussed in, e.g., Refs. [78-80], while statistical arguments relying on the existence of the discretuum have been used, e.g., in Refs. $[77,81,40]$ in tuning the cosmological constant.

Although this is a bit far from the concrete goal of our paper, it is worth discussing how this discretuum may be expected to arise in the heterotic theory. In type II theories, as in M-theory, the discretuum is populated by vacua with various quantized values of the RR and NS fluxes, and with different wrapped branes, consistent with the tadpole conditions arising from the Gauss's law constraints on the various $p$-form field strengths. In the heterotic theory, there are a few quantum numbers which contribute to the large number of vacua. In addition to the large number of choices of vector bundles on a fixed manifold [characterized by the topological numbers $c_{2}\left(V_{i}\right), c_{3}\left(V_{i}\right)$, for instance], there are also background NS fluxes. Finally, there is the possibility of nonKählerity, which is roughly dual to the possibilities of different fluxes in type II theories [41].

As described at length in Ref. [78], to get a good handle on this large set of possibilities, it will probably be necessary to find auxiliary ensembles which accurately model the space of vacua. We have little to say about this at present but leave it as an ambitious goal for future research.

\section{ACKNOWLEDGMENTS}

We would like to thank B. Acharya, N. Arkani-Hamed, P. Aspinwall, M. Becker, K. Dasgupta, M. Dine, M. Douglas, R. Kallosh, A. Krause, E. Silverstein, S. Thomas, S. Trivedi, and $\mathrm{E}$. Witten for interesting discussions on related subjects. S.G. is supported in part by the RFBR Grant No. 01-0100549 and the RFBR grant for Young Scientists 02-0106322. The work of S.K. is supported in part by the David and Lucile Packard Foundation, National Science Foundation Grant No. PHY-0097915, and the DOE under Contract No. DE-AC03-76SF00515. The work of L.M. is supported by the National Science Foundation.
[1] P. Candelas, G. Horowitz, A. Strominger, and E. Witten, Nucl. Phys. B258, 46 (1985).

[2] G. Coughlan, W. Fischler, E. Kolb, S. Raby, and G. Ross, Phys. Lett. 131B, 59 (1983); B. de Carlos, J. A. Casas, F. Quevedo, and E. Roulet, Phys. Lett. B 318, 447 (1993); T. Banks, D. B. Kaplan, and A. E. Nelson, Phys. Rev. D 49, 779 (1994)

[3] M. Dine, R. Rohm, N. Seiberg, and E. Witten, Phys. Lett. 156B, 55 (1985).

[4] J. P. Derendinger, L. E. Ibanez, and H. P. Nilles, Phys. Lett. 155B, 65 (1985).

[5] R. Rohm and E. Witten, Ann. Phys. 170, 454 (1986).

[6] A. Niemeyer and H. P. Nilles, "Gaugino condensation and the vacuum expectation value of the dilaton," hep-th/9508173.

[7] H. P. Nilles, "Dynamical gauge coupling constants," hep-ph/9601241.

[8] P. Binétruy, M. K. Gaillard, and Y.-Y. Wu, Nucl. Phys. B481, 109 (1996).

[9] T. Barreiro, B. de Carlos, and E. J. Copeland, Phys. Rev. D 58, 083513 (1998).

[10] Y.-Y. Wu, Nucl. Phys. B481, 109 (1996).

[11] R. Xiu, "Supersymmetry breaking scheme and the derivation of $M_{\mathrm{GUT}}=10^{16} \mathrm{GeV}$ from a string model," hep-ph/9412262.

[12] E. Witten, Nucl. Phys. B258, 75 (1985).
[13] E. Witten, "Deconstruction, $G_{2}$ holonomy, and doublet-triplet splitting," hep-ph/0201018.

[14] G. Cardoso, G. Curio, G. Dall'Agata, and D. Lüst, "Heterotic string theory on non-Kaehler manifolds with H-flux and gaugino condensate," hep-th/0310021.

[15] K. Becker, M. Becker, K. Dasgupta, P. Green, and E. Sharpe, "Compactifications of heterotic strings on non-Kähler complex manifolds: II," hep-th/0310058.

[16] E. I. Buchbinder and B. A. Ovrut, "Vacuum stability in heterotic M-theory," hep-th/0310112.

[17] E. Bergshoeff, M. de Roo, B. de Wit, and P. van Nieuwenhuizen, Nucl. Phys. B195, 97 (1982).

[18] J. Polchinski, Superstring Theory and Beyond, Vol. II of String Theory (Cambridge University Press, Cambridge, UK, 1998).

[19] M. A. Shifman and A. I. Vainshtein, Nucl. Phys. B296, 445 (1988).

[20] M. A. Shifman and A. I. Vainshtein, Nucl. Phys. B359, 571 (1991).

[21] N. Seiberg, Phys. Rev. D 49, 6857 (1994); K. Intriligator, R. G. Leigh, and N. Seiberg, ibid. 50, 1092 (1994).

[22] D. Finnell and P. Pouliot, "Instanton calculations versus exact results in 4 dimensional SUSY gauge theories," hep-th/9503115.

[23] S. Gukov, C. Vafa, and E. Witten, Nucl. Phys. B584, 69 (2000). 
[24] S. Gukov, Nucl. Phys. B574, 169 (2000).

[25] M. Becker and D. Constantin, J. High Energy Phys. 08, 015 (2003).

[26] K. Behrndt and S. Gukov, Nucl. Phys. B580, 225 (2000).

[27] V. Kaplunovsky and J. Louis, Nucl. Phys. B422, 57 (1994).

[28] R. McLean, Comm. Anal. Geom. 6, 705 (1998).

[29] A. Strominger, S. T. Yau, and E. Zaslow, Nucl. Phys. B479, 243 (1996).

[30] R. Gopakumar and C. Vafa, Adv. Theor. Math. Phys. 2, 399 (1998).

[31] P. S. Aspinwall and C. A. Lutken, Nucl. Phys. B353, 427 (1991); ibid. B355, 482 (1991).

[32] H. Nishi, Int. J. Math 9, 295 (1998).

[33] D. Auckly, Math. Proc. Cambridge Philos. Soc. 115, 229 (1994).

[34] L. Rozansky, Commun. Math. Phys. 171, 279 (1995).

[35] E. Witten, in Symposium on Anomalies, Geometry, Topology, edited by W. Bardeen and A. White (World Scientific, Singapore, 1985).

[36] J. O. Conrad, J. High Energy Phys. 11, 022 (2000).

[37] E. Witten, J. High Energy Phys. 02, 030 (2000).

[38] A. Strominger, Nucl. Phys. B274, 253 (1986).

[39] See, e.g., B. R. Greene, K. Kirklin, P. Miron, and G. G. Ross, Nucl. Phys. B278, 667 (1986); D. Gepner, "String theory on Calabi-Yau manifolds: The three generations case," hep-th/9301089; S. Kachru, Phys. Lett. B 349, 76 (1995); B. Ovrut, T. Pantev, and R. Reinbacher, J. High Energy Phys. 05, 040 (2003).

[40] S. Kachru, R. Kallosh, A. Linde, and S. Trivedi, Phys. Rev. D 68, 046005 (2003).

[41] K. Dasgupta, G. Rajesh, and S. Sethi, J. High Energy Phys. 08, 023 (1999); K. Becker and K. Dasgupta, ibid. 11, 006 (2002); S. Gurrieri, J. Louis, and A. Micu, Nucl. Phys. B654, 61 (2003); G. L. Cardoso, G. Curio, G. Dall'Agata, D. Lüst, P. Manousselis, and G. Zoupanos, ibid. B652, 5 (2003); S. Kachru, M. Schulz, P. Tripathy, and S. Trivedi, J. High Energy Phys. 03, 061 (2003); K. Becker, M. Becker, K. Dasgupta, and P. Green, ibid. 04, 007 (2003).

[42] K. Becker, M. Becker, K. Dasgupta, and S. Prokushkin, Nucl. Phys. B666, 149 (2003).

[43] G. L. Cardoso, G. Curio, G. Dall'Agata, and D. Lust, J. High Energy Phys. 10, 004 (2003).

[44] M. Bershadsky, S. Cecotti, H. Ooguri, and C. Vafa, "KodairaSpencer theory of gravity and exact results for quantum string amplitudes," hep-th/9309140.

[45] M. Atiyah, J. Maldacena, and C. Vafa, J. Math. Phys. 42, 3209 (2001)

[46] See, e.g., D. Morrison and C. Vafa, Nucl. Phys. B473, 74 (1996); B476, 437 (1996); N. Seiberg and E. Witten, "Comments on string dynamics in six dimensions," hep-th/9603003.

[47] E. Witten, "Strong coupling expansion of Calabi-Yau compactification," hep-th/9602070.

[48] E. Witten, Nucl. Phys. B403, 159 (1993).

[49] P. Aspinwall, B. Greene, and D. Morrison, Nucl. Phys. B416, 414 (1994)

[50] S. Kachru and C. Vafa, Nucl. Phys. B450, 69 (1995); S. Ferrara, J. Harvey, A. Strominger, and C. Vafa, ibid. B361, 59 (1995).
[51] J. Louis, J. Sonnenschein, S. Theisen, and S. Yankielowicz, Nucl. Phys. B480, 185 (1996).

[52] R. Schimmrigk, Phys. Lett. B 388, 60 (1996).

[53] B. de Carlos, J. A. Casas, and C. Munoz, Nucl. Phys. B399, 623 (1993)

[54] M. Atiyah and E. Witten, Adv. Theor. Math. Phys. 6, 1 (2003).

[55] B. Acharya and E. Witten, "Chiral fermions from manifolds of $G_{2}$ holonomy," hep-th/0109152.

[56] T. Friedmann and E. Witten, "Unification scale, proton decay, and manifolds of $G_{2}$ holonomy," hep-th/0211269.

[57] I. R. Klebanov and E. Witten, Nucl. Phys. B664, 3 (2003).

[58] B. S. Acharya, "A moduli fixing mechanism in M theory," hep-th/0212294.

[59] M. Cvetic, G. Shiu, and A. M. Uranga, Nucl. Phys. B615, 3 (2001).

[60] M. Cvetic, P. Langacker, and J. Wang, Phys. Rev. D 68, 046002 (2003)

[61] M. Cvetic and I. Papadimitriou, Phys. Rev. D 68, 046001 (2003).

[62] S. Gukov, S. T. Yau, and E. Zaslow, "Duality and fibrations on G(2) manifolds," hep-th/0203217.

[63] H. Ita, Y. Oz, and T. Sakai, J. High Energy Phys. 04, 001 (2002).

[64] T. Friedmann, Nucl. Phys. B635, 384 (2002).

[65] E. Witten, "Anomaly cancellation on manifolds of $G_{2}$ holonomy," hep-th/0108165.

[66] J. de Boer, R. Dijkgraaf, K. Hori, A. Keurentjes, J. Morgan, D. R. Morrison, and S. Sethi, Adv. Theor. Math. Phys. 4, 995 (2002).

[67] A. Keurentjes and S. Sethi, Phys. Rev. D 66, 046001 (2002).

[68] D. M. Austin, J. Diff. Geom. 32, 383 (1990).

[69] R. Fintushel and R. Stern, J. Lond. Math. Soc. 150, 125 (1989).

[70] R. Fintushel and T. Lawson, Topol. Appl. 23, 305 (1986).

[71] E. Witten, Nucl. Phys. B268, 79 (1986).

[72] E. Silverstein and E. Witten, Nucl. Phys. B444, 161 (1995); A. Basu and S. Sethi, Phys. Rev. D 68, 025003 (2003); C. Beasley and E. Witten, J. High Energy Phys. 10, 065 (2003).

[73] G. Moore, G. Peradze, and N. Saulina, Nucl. Phys. B607, 117 (2001); E. Buchbinder, R. Donagi, and B. Ovrut, ibid. B653, 400 (2003); G. Curio and A. Krause, ibid. B643, 131 (2002).

[74] C. P. Burgess, R. Kallosh, and F. Quevedo, J. High Energy Phys. 10, 056 (2003).

[75] E. Silverstein, "(A)dS backgrounds from asymmetric orientifolds," hep-th/0106209.

[76] C. Escoda, M. Gomez-Reino, and F. Quevedo, J. High Energy Phys. 11, 065 (2003); A. Frey, M. Lippert, and B. Williams, Phys. Rev. D 68, 046008 (2003).

[77] R. Bousso and J. Polchinski, J. High Energy Phys. 06, 006 (2000).

[78] M. Douglas, J. High Energy Phys. 05, 046 (2003); S. Ashok and M. Douglas, "Counting flux vacua," hep-th/0307049.

[79] L. Susskind, "The anthropic landscape of string theory," hep-th/0302219.

[80] B. Acharya, "Compactification with flux and yukawa hierarchies," hep-th/0303234.

[81] J. Feng, J. March-Russell, S. Sethi, and F. Wilczek, Nucl. Phys. B602, 307 (2001). 